\title{
La heterogeneidad de los efectos de la educación preescolar sobre los resultados cognitivos en América Latina
}

\author{
Juan Antonio Dip y Luis Fernando Gamboa
}

\section{Resumen}

En este estudio se utiliza un modelo de emparejamiento por puntaje de propensión (propensity score matching) para cuantificar la importancia que tiene la educación preescolar en los resultados académicos en el corto y mediano plazo en varios países de América Latina, utilizando la información proveniente de Tercer Estudio Regional Comparativo y Explicativo (TERCE) para tercer y sexto grado. Los resultados son heterogéneos a nivel de país y de grado escolar y fluctúan entre 0,05 y 0,3 desviaciones estándar. En tercer grado, al menos diez países presentan un efecto superior a 0,10 desviaciones estándar en Lectura y Matemáticas, mientras para sexto grado son siete países los que presentan este efecto en las tres pruebas evaluadas.

\section{Palabras clave}

Educación preescolar, niños, indicadores educativos, desarrollo del niño, rendimiento escolar, América Latina

\section{Clasificación JEL}

$$
\text { C14, H4, } 121
$$

\section{Autores}

Juan Antonio Dip, Doctor en Economía, Facultad de Ciencias Económicas, Universidad Nacional de Misiones (Argentina). Correo electrónico: dip@fce.unam.edu.ar.

Luis Fernando Gamboa, Doctor en Economía, Departamento de Economía, Universidad Jorge Tadeo Lozano (Colombia). Correo electrónico: luisfw.gamboan@utadeo.edu.co. 


\section{Introducción}

La importancia de evaluar los logros de las políticas educativas es indiscutible y cuando se trata de etapas tempranas el margen de maniobra y la eficacia de los recursos puede ser mucho mayor en términos económicos (Heckman, Pinto y Savelyev, 2013). El objetivo de este estudio es identificar el efecto de la educación preescolar en el desempeño de estudiantes de tercer y sexto grado en las pruebas de Ciencias, Matemáticas y Lectura del Tercer Estudio Regional Comparativo y Explicativo (TERCE) llevadas a cabo en 2013. Dicho estudio forma parte de una serie de estudios regionales conocidos como Segundo Estudio Regional Comparativo y Explicativo (SERCE) y Primer Estudio Regional Comparativo y Explicativo (PERCE) ${ }^{1}$, que son comparables entre sí y entre países gracias a la metodología estadística y al método de selección muestral estratificado, por conglomerados y bietápico.

Este tema es crucial por la preocupación institucional de mejorar las condiciones de vida de los latinoamericanos, resultante de los bajos niveles de capital humano promedio en la región, pero también de la necesidad de mejorar la equidad en estos países. Recientemente, las diferencias en materia de logros cognitivos de estos países con respecto a los más desarrollados se han hecho visibles en los resultados del Programa para la Evaluación Internacional de los Alumnos (PISA) y en las pruebas Tendencias en el Estudio Internacional de Matemáticas y Ciencias (TIMMS). La inversión en programas de educación preescolar es un mecanismo que tiene efectos positivos directos e indirectos. Por un lado, potencializa las capacidades de los niños a un costo menor al que tendría si se hace en poblaciones mayores y, por el otro, reduce las barreras de participación laboral de las madres en la economía (Cascio, 2015; Shah y otros, 2017; Heckman, Pinto y Savelyev, 2013; Loeb y otros, 2007; Behrman, Cheng y Todd, 2004; Skibbe y otros, 2011; Shonkoff y Phillips, 2000).

Estos efectos persisten en el tiempo y otros decrecen, pero se mantienen aún varios años después (Nores y Barnett, 2010; Berlinski, Galiani y Manacorda, 2008; Magnuson, Ruhm y Waldfogel, 2007a, 2007b). En la literatura mencionada se coincide en que existen efectos positivos, aunque moderados, que pueden permanecer en los niños en el tiempo y, por lo tanto, es un área de obligada intervención y monitoreo entre países (UNESCO, 2014).

Según la Organización de las Naciones Unidas para la Educación, la Ciencia y la Cultura (UNESCO) (2013), la tasa neta de matrícula en educación preprimaria promedio pasó del 55,5\% al 66\% entre 1990 y 2010. Sin embargo, la cobertura preprimaria tiene gran heterogeneidad entre estos países (40\% a 90\%). Por ejemplo, México y el Uruguay duplican la tasa de matrícula de Bolivia (Estado Plurinacional de) y el Paraguay en 2010. En 2014, la tasa de cobertura neta de preescolar ha aumentado y en países como el Perú y el Uruguay se encuentran por encima del $85 \%$, aunque el avance no sea significativo para otras economías como las centroamericanas (Costa Rica, Guatemala, Honduras, y Panamá) que se encuentran por debajo del 53\%.

No obstante, la asistencia a preescolar, al ser un servicio educativo, enfrenta varios retos. Por un lado, no existe un estándar de calidad que permita hacer del todo comparable el conjunto de acciones y la composición de insumos utilizados en su prestación. Por otro lado, la permanencia del estudiante en años lectivos depende tanto de factores de oferta como de demanda.

En América Latina existe poca evidencia sobre efectos causales del preescolar con pruebas comparables a nivel internacional. Existe literatura reciente que provee una descripción detallada de los problemas de acceso y de cobertura de la educación inicial en América Latina, como los estudios del Programa Regional sobre Políticas Sociales (SOPLA) (2014) y Gamboa y Krüger (2016) quienes presentan un panorama sobre las diferencias en los indicadores de cobertura a nivel de países que muestran las condiciones adversas de las poblaciones más vulnerables en acceso. Estos últimos utilizan una

\footnotetext{
1 La comparación es posible entre TERCE y SERCE. Véanse detalles sobre el desarrollo histórico de esta prueba en http://www. unesco.org.
} 
descomposición no paramétrica de los resultados de la prueba PISA 2012 para mostrar la contribución de la educación inicial en Lectura y Matemáticas en la Argentina, el Brasil, Chile, Colombia, México, el Perú y el Uruguay. En dicho trabajo hay indicios de segregación por nivel socioeconómico en el acceso y brechas en puntajes mientras más tiempo se asiste a una institución de educación preescolar.

En estos estudios, existe el hallazgo común de que los países latinoamericanos tienen altos niveles de retraso en niños pobres cuando comienzan la escolaridad formal, que persisten en el tiempo (Schady y otros, 2015). Araujo, Dormal y Schady (2017) ofrece una discusión interesante sobre los hallazgos en materia de calidad en los jardines de cuidado infantil y provee una extensa revisión de la literatura sobre el tema.

Para el caso de Chile, el Ministerio de Educación (MINEDUC, 2014) revela estudios del impacto de corto, mediano y largo plazo que tiene la asistencia de los niños a centros de educación parvularia. En términos generales, encuentran que asistir a prekínder y a kínder tiene efectos positivos y significativos, aunque resalta que "no se cuenta con evidencia concluyente para determinar la universalidad del impacto de la asistencia a centros de educación parvularia en el desarrollo de los niños de manera integral" (pág. 22).

Otros estudios como Bernal y otros (2012) y Bernal y Camacho (2012) van más allá y proveen evidencia en relación con el impacto causal de programas de educación inicial sobre los resultados de los niños. Un estudio de Bernal y otros (2015) muestra que las ganancias en calidad son nulas por cambios de modelos de enseñanza de cuidado comunitario a centros grandes con capacidad para 150 niños o más cada uno en Colombia. Otros estudios más detallados estiman que el efecto puede estar en alrededor de un 0,20 de una desviación estándar en competencias cognitivas (véase Berlinski y Schady, 2015). La contribución del presente estudio es múltiple. En primer lugar, como se mencionó anteriormente, insiste en la necesidad de encontrar respuestas a las diferencias de rendimiento en pruebas posteriores en el ciclo educativo como las pruebas PISA. En segundo lugar, aporta evidencia sobre los efectos cognitivos de incrementar los niveles de acceso a educación inicial luego de controlar por otras variables asociadas a los estudiantes, sus familias, sus colegios y sus sistemas educativos. En tercer lugar, llama la atención sobre la necesidad de trabajar de manera conjunta para aprender de las diferencias con países vecinos en términos de iniciativas de cobertura. No obstante estos aspectos, la interpretación de los resultados debe ser cuidadosa, porque existen factores no siempre observables que pueden influir en las conclusiones.

La estrategia empírica utiliza un modelo de emparejamiento por puntaje de propensión (propensity score matching) que reduce el sesgo proveniente de la no observabilidad de algunos factores.

Los resultados son heterogéneos pero positivos en los países participantes en el TERCE. En los países centroamericanos parece haber evidencia de impactos más grandes en el puntaje de la prueba que en los demás países, pero no se encuentra un patrón de aumento o disminución del efecto entre países entre tercer y sexto grado.

Este artículo está dividido en cinco secciones. La segunda sección presenta la estrategia empírica para comparar los resultados de diferentes niveles de exposición a un programa preescolar. La tercera sección describe los datos utilizados para la evaluación del efecto. La cuarta sección presenta los resultados y la última sección resume algunas recomendaciones para tener en consideración.

\section{Estrategia empírica}

El puntaje estandarizado en una prueba cognitiva (Matemáticas, Ciencias o Lectura) del individuo $i$ en el colegio $s$ en el municipio $m$ en el país $j\left(Y_{i s m j}\right)$ ( depende de factores asociados al individuo y su familia $(X)$, variables del plantel educativo $(Z)$ y variables de la región o país $(W)$. 


$$
Y_{i s m j}=a+\operatorname{Pre}_{i j}+\beta_{1} X+\beta_{2} Z+\beta_{3} W+\varepsilon_{i}
$$

Entre las variables consideradas dentro del vector $X$ están la edad, el género, el nivel de riqueza del hogar y el nivel educativo de los padres. Los controles de colegio (Z) incluyen, si el colegio es urbano o rural y si su gestión es estatal o privada y, adicionalmente, se introduce un indicador de calidad del plantel obtenido a partir del clima escolar en el aula de clase. No obstante esto, no se controla por la motivación de los padres o la habilidad del estudiante.

Si se asume que estas variables están distribuidas de manera aleatoria, es decir $E(X / \varepsilon)=0$, el efecto del preescolar podría estimarse por un modelo de mínimos cuadrados ordinarios.

Sin embargo, padres más motivados pueden incentivar mayores y mejores prácticas educativas en casa y, por tanto, se estaría sobreestimando el efecto del preescolar. Si la correlación entre la habilidad y el desempeño es positiva y la correlación entre habilidad y motivación de los padres hacia la educación también es positiva, el estimador ( Pre $_{i j}$ ) será mayor al verdadero. Por otro lado, el método de mínimos cuadrados ordinarios puede desconocer la existencia de un impacto heterogéneo del tratamiento, en este caso está dado por la asistencia a preescolar (Black, 2015). Para controlar por estas limitaciones se utiliza un método cuasiexperimental de evaluación de efecto causal como el emparejamiento por puntaje de propensión.

El emparejamiento por puntaje de propensión, propuesto por Rosenbaum y Rubin (1983) permite corregir el problema de sesgo de selección cuando se piensa que los niños de familias con mejores condiciones socioeconómicas tienden a comenzar la escuela en forma temprana y con una mayor posibilidad de concurrir a un establecimiento de educación infantil. Es decir, asistir a la educación inicial depende de las decisiones de los padres y, por ende, no es aleatoria. Por ello, las características medibles y no medibles de los niños están asociadas con la probabilidad de asistir al preescolar (tratamiento) y con el resultado (pruebas estandarizadas). El emparejamiento por puntaje de propensión asume que hay un conjunto de $X$ covariables observables, tal que, después de controlar por ellas, los resultados potenciales son independientes del estatus de tratamiento (independencia condicional) y que para cada valor de $X$, existe una probabilidad positiva de ser a la vez tratado y no tratado (soporte común).

La estrategia planteada estima el efecto causal de asistir a educación inicial en el logro académico (medido a través de distintas pruebas estandarizadas del TERCE) y, en especial el efecto promedio del tratamiento sobre el tratado (average treatment effect on the treated). De acuerdo con Heckman y Robb (1986), estimar este promedio es valioso para responder preguntas de investigación que conciernen a la elaboración de políticas sociales, lo que sería muy útil en el caso de una política educacional que tenga como objetivo extender la educación preescolar obligatoria.

Es necesario identificar el tratamiento, el resultado y las covariables intervinientes (véanse más detalles en Caliendo y Kopeinig, 2008, y Stuart, 2010). En este caso, la variable de tratamiento ha sido PREKFOR6 que toma el valor 1 si el alumno ha asistido a la educación inicial entre los 4 y 6 años. El valor 0 en caso de que no haya asistido. La variable de resultado es el puntaje z-score (media igual a 0 y desvío estándar igual a 1), obtenido por el alumno en Lectura y Matemáticas en tercer grado, y adicionalmente Ciencias en sexto grado para los países participantes².

La selección de las covariables para el emparejamiento podría realizarse de acuerdo a su relacionamiento con el tratamiento y con el resultado (Stuart, 2010), sin embargo Brookhart y otros (2006) sugieren que se deberían incluir en el puntaje de propensión, variables que estén relacionadas más con el resultado que con el tratamiento ya que esto permite aumentar la precisión del efecto estimado de exposición al tratamiento sin incrementar el sesgo. Teniendo en cuenta esto y la literatura

\footnotetext{
2 Aunque la prueba presenta una escala de puntaje con media 700, la variable de resultado se ha reescalado para poder interpretar los resultados en términos de desviaciones estándar.
} 
existente sobre educación preescolar, las covariables incluidas son las consideradas en el modelo de mínimos cuadrados ordinarios, incluyéndose las expectativas de los padres.

Luego, la elección de la técnica de emparejamiento se realizó en base a la diferencia estandarizada de las medias de las covariables. Para el cálculo del efecto promedio del tratamiento sobre el tratado se ha seleccionado la estrategia de Kernel dado que la diferencia ha sido menor en comparación con las otras técnicas probadas. Para todos los modelos (asignaturas y para cada uno de los países), se verificaron dos indicadores propuestos por Rubin (2001) con el fin de determinar el balance logrado con el puntaje de propensión: B, que representa la diferencia estandarizada de las medias del índice lineal de dicho puntaje en el grupo de tratados y no tratados pertenecientes a la muestra apareada, y R, que es la razón de la varianza del puntaje de propensión en la muestra tratada y sin tratar. Rubin (2001) sugiere que un valor de B menor a 0,25 indica que las mismas están balanceadas. La razón de varianza $\mathrm{R}$ debe tender al valor 1 pero valores entre 0,5 y 2 se consideran aceptables (véase el cuadro 5).

En todos los ejercicios realizados, los valores obtenidos para B han sido menores a 0,25 y los de R se aproximaron al valor de 1. En este tipo de metodología, la interpretación del efecto promedio del tratamiento sobre el tratado encontrados depende de los errores estándar que se calculan. Si bien la literatura se ha concentrado en ajustar los errores estándar mediante el método de remuestreo (bootstrap), Abadie e Imbens (2005) demuestran que los errores calculados por el método de remuestreo para el caso de que se aplique el criterio de emparejamiento del vecino más cercano (nearest neighbor matching) no serían válidos. Sin embargo, para el caso especial del método de Kernel, esto no es concluyente, ya que las discontinuidades que surgen en el emparejamiento del vecino más cercano no se observan en este caso (Wagstaff, 2007).

La generalización de resultados de estimaciones obtenidas a partir de muestras de datos requiere el uso de pesos muestrales, porque ignorarlos en el emparejamiento por puntaje de propensión limita la validez externa de los resultados ya que las inferencias realizadas sobre la población se basan en una muestra no representativa. Por ello, los pesos muestrales y el diseño de la muestra hacen parte del proceso de estimación cuando se estima el puntaje de propensión y cuando se utiliza ese puntaje para estimar el efecto de tratamiento. De esta manera se distinguen dos efectos de tratamiento en el tratado: el efecto promedio del tratamiento sobre el tratado de la muestra, donde los pesos muestrales se incorporan en el cálculo del puntaje de propensión únicamente y el efecto promedio del tratamiento sobre el tratado de la población objetivo, en el cual los pesos muestrales (además de incorporarse al puntaje de propensión), son combinados con los pesos de la estrategia de emparejamiento elegida para redefinir un nuevo peso que es utilizado en una regresión ponderada (DuGoff, Schuler y Stuart, 2014).

\section{Datos}

EI TERCE se realizó bajo coordinación de la UNESCO en 2013 para 15 países latinoamericanos (Argentina, Brasil, Chile, Colombia, Costa Rica, Ecuador, Guatemala, Honduras, México, Nicaragua, Panamá, Paraguay, Perú, República Dominicana y Uruguay) con la participación de más de 100.000 estudiantes latinoamericanos. Esta iniciativa evalúa el desempeño escolar de Matemáticas, Lenguaje (lectura y escritura) en tercer grado, e incluye Ciencias Naturales para sexto grado. Para poder hacer comparaciones a nivel de país y a lo largo del tiempo con el SERCE 2006, la prueba TERCE implementó un método de selección muestral estratificado, por conglomerados y bietápico. En la primera etapa se seleccionaron escuelas (en cada estrato explícito) con probabilidad de selección proporcional al tamaño. En la segunda etapa se seleccionó aleatoriamente un aula y se aplicó a todos los alumnos que pertenecían a esa aula. Esta técnica muestral permite hacer inferencias a nivel del sistema educativo al reconocer la existencia de diferentes niveles de variabilidad (intra y entre) 
escuelas $^{3}$ (pueden verse más detalles sobre la representatividad de la muestra en UNESCO, 2016). En el cuadro A1.1 se puede ver que los tamaños de la muestra son considerables para cada uno de los países participantes una vez que se tienen en cuenta los pesos muestrales aportados por el TERCE (en Lectura para tercer grado hubo más de 2.600 estudiantes en el país con una muestra de menor tamaño absoluto). La elaboración de las pruebas cognitivas incluyó una revisión de los marcos curriculares de los países participantes para poder establecer los niveles de desempeño por áreas. En consecuencia, la utilización de los contenidos comunes y un diseño muestral similar al implementado por otras pruebas como PISA o el Estudio Internacional del Progreso en Competencia Lectora (PIRLS), le permite al TERCE contribuir al enriquecimiento de la literatura sobre evaluación educativa en la región. La variable que permite la identificación de quienes asistieron a preescolar es creada por el TERCE y se refiere a quienes asistieron entre los 4 y 6 años de edad.

Antes de considerar los resultados globales obtenidos por los países participantes, conviene dar una mirada a los indicadores agregados de la situación del sector educativo y su bienestar actual (véase el cuadro 1). América Latina ha venido avanzando en la reducción de la pobreza, pero aún hay países que tienen una incidencia mayor al 10\%, como Colombia, el Ecuador, El Salvador, Honduras y Nicaragua, mientras que otros como la Argentina, Chile y Costa Rica tienen niveles por debajo del $5 \%$. La distribución geográfica de la población muestra países muy urbanos como la Argentina o el Uruguay y otros con una ruralidad alta, más común en los países centroamericanos, como Guatemala (48\%) y Honduras (45\%).

Cuadro 1

Indicadores socioeconómicos

(En porcentajes)

\begin{tabular}{|c|c|c|c|c|c|c|c|}
\hline \multirow[t]{2}{*}{ País } & \multirow{2}{*}{$\begin{array}{c}\text { Pobreza } \\
\text { (tasa de incidencia, } \\
\text { en porcentajes) }\end{array}$} & \multirow{2}{*}{$\begin{array}{c}\text { Ruralidad } \\
\text { (en porcentajes } \\
\text { sobre población total) }\end{array}$} & \multirow{2}{*}{$\begin{array}{c}\text { Gasto público } \\
\text { en educación } \\
\text { (en porcentajes } \\
\text { del PBI })^{\star \star}\end{array}$} & \multicolumn{4}{|c|}{$\begin{array}{l}\text { Participación (tasa neta de escolarización) } \\
\text { (en porcentajes) })^{\star \star \star}\end{array}$} \\
\hline & & & & Preprimaria & Primaria & Secundaria & Terciaria \\
\hline Argentina & 4,3 & 8 & 5,33 & 72,47 & 99,3 & 88,2 & 82,9 \\
\hline Brasil & 7,6 & 14 & 5,99 & 81,98 & 92,7 & 81,3 & 50,6 \\
\hline Chile & 2,0 & 10 & 4,92 & 80,76 & 94,3 & 87,9 & 88,5 \\
\hline Colombia & 13,2 & 24 & 4,49 & 78,29 & 90,6 & 78,2 & 55,6 \\
\hline Costa Rica & 3,9 & 23 & 7,18 & 50,36 & 96,4 & 79,3 & 53,6 \\
\hline Ecuador & 10,2 & 36 & 4,96 & 66,14 & 91,2 & 85,4 & 40,4 \\
\hline El Salvador & 11,3 & 33 & 3,55 & 42,94 & 91,2 & 68,7 & 55,5 \\
\hline Guatemala & 24,0 & 48 & 2,96 & 41,91 & 85,4 & 48,1 & 29,1 \\
\hline Honduras & 31,2 & 45 & 5,87 & 68,72 & 93,0 & 49,4 & 21,8 \\
\hline México & 11,0 & 21 & 5,31 & - & 95,0 & 90,5 & 22,0 \\
\hline Nicaragua & 17,1 & 41 & 4,49 & 46,83 & 96,9 & 48,9 & 29,9 \\
\hline Perú & 9,0 & 21 & 9,00 & 88,36 & 94,0 & 77,6 & - \\
\hline República Dominicana & 9,1 & 21 & - & 43,16 & 86,89 & 66,0 & 50,12 \\
\hline Uruguay & 1,3 & 5 & 4,36 & 88,23 & 94,2 & 76,3 & - \\
\hline
\end{tabular}

Fuente: Elaboración propia, sobre la base de información de la Organización de las Naciones Unidas para la Educación, la Ciencia y la Cultura (UNESCO)

Nota: * Sobre la base de 3,10 dólares PPP por día; ** Último dato educativo disponible en el Instituto de Estadística de la UNESCO; ${ }^{* \star \star}$ La información sobre participación en sistema educativo corresponde a 2015 excepto para la Argentina, Honduras, Nicaragua (2010) y Panamá (2014).

\footnotetext{
3 Además se dispone de los pesos de senado, que consisten en una reponderación del peso muestral originalmente calculado en cada país, de manera que las poblaciones representadas sean del mismo tamaño en cada uno de ellos. Permiten realizar estimaciones o análisis comparativos entre países independientemente del tamaño poblacional de estos.
} 
Los niveles de gasto público en educación no son considerablemente diferentes, pero es posible establecer un conjunto de países que gastan más del $5 \%$ del PIB en educación (siete países en los que sobresale el Perú para el período reciente) y otros países con menor gasto, siendo Guatemala el que menor porcentaje de recursos dedica al sector educativo.

La participación es altamente heterogénea en los niveles superiores: sobresalen la Argentina y Chile con participaciones superiores al $83 \%$ en todos los niveles y México, que tiene cobertura bastante alta en educación primaria y secundaria, pero decae en la superior. En el lado opuesto se ubican Guatemala y Honduras, cuyas tasas de participación están por debajo del 50\% en educación secundaria y terciaria.

En relación con la clasificación de desempeño entre áreas y países (véase el cuadro 2), las diferencias entre el país de mayor desempeño (Chile) y menor desempeño (República Dominicana) superan los 185 puntos en tercer grado y se reducen para sexto grado. Costa Rica y México se encuentran en los niveles superiores de desempeño y, en el lado opuesto, el Paraguay acompaña a la República Dominicana en la parte baja de la clasificación. Los resultados en cada país tienen niveles de heterogeneidad variable, es decir, alta dispersión (Colombia) y baja dispersión (Nicaragua).

Cuadro 2

Puntaje promedio en el Tercer Estudio Regional Comparativo y Explicativo (TERCE) por área y país

\begin{tabular}{|c|c|c|c|c|c|c|c|c|c|c|}
\hline \multirow{3}{*}{ País } & \multicolumn{4}{|c|}{ Lectura } & \multicolumn{4}{|c|}{ Matemáticas } & \multirow{2}{*}{\multicolumn{2}{|c|}{$\begin{array}{c}\text { Ciencias } \\
\text { Sexto grado }\end{array}$}} \\
\hline & \multicolumn{2}{|c|}{ Tercer grado } & \multicolumn{2}{|c|}{ Sexto grado } & \multicolumn{2}{|c|}{ Tercer grado } & \multicolumn{2}{|c|}{ Sexto grado } & & \\
\hline & Promedio & $\begin{array}{l}\text { Desviación } \\
\text { estándar }\end{array}$ & Promedio & $\begin{array}{l}\text { Desviación } \\
\text { estándar }\end{array}$ & Promedio & $\begin{array}{l}\text { Desviación } \\
\text { estándar }\end{array}$ & Promedio & $\begin{array}{l}\text { Desviación } \\
\text { estándar }\end{array}$ & Promedio & $\begin{array}{l}\text { Desviación } \\
\text { estándar }\end{array}$ \\
\hline Argentina & 703 & 4,89 & 707 & 4,50 & 717 & 4,83 & 722 & 4,14 & 700 & 4,65 \\
\hline Brasil & 712 & 4,99 & 721 & 4,91 & 727 & 6,05 & 709 & 5,29 & 700 & 4,52 \\
\hline Chile & 802 & 3,96 & 776 & 3,23 & 787 & 4,04 & 793 & 4,24 & 768 & 4,63 \\
\hline Colombia & 714 & 8,33 & 726 & 5,49 & 694 & 7,80 & 705 & 5,45 & 733 & 4,57 \\
\hline Costa Rica & 754 & 3,24 & 755 & 2,80 & 750 & 2,86 & 730 & 3,09 & 756 & 3,14 \\
\hline Ecuador & 698 & 4,72 & 683 & 5,14 & 703 & 4,75 & 702 & 4,64 & 711 & 4,57 \\
\hline Guatemala & 678 & 3,87 & 678 & 3,20 & 672 & 3,28 & 672 & 2,96 & 684 & 3,43 \\
\hline Honduras & 681 & 4,14 & 662 & 6,19 & 680 & 4,97 & 661 & 4,01 & 668 & 3,52 \\
\hline México & 718 & 3,25 & 735 & 3,34 & 741 & 3,26 & 768 & 3,51 & 732 & 3,23 \\
\hline Nicaragua & 654 & 2,84 & 662 & 2,72 & 653 & 3,07 & 643 & 2,44 & 668 & 3,38 \\
\hline Paraguay & 653 & 4,81 & 652 & 3,99 & 652 & 5,42 & 641 & 3,75 & 646 & 4,12 \\
\hline Perú & 719 & 3,91 & 703 & 3,39 & 716 & 4,10 & 721 & 3,92 & 701 & 3,61 \\
\hline República Dominicana & 614 & 3,50 & 633 & 3,29 & 602 & 3,68 & 622 & 2,31 & 632 & 3,01 \\
\hline Uruguay & 728 & 7,15 & 736 & 5,02 & 742 & 7,96 & 765 & 6,38 & 725 & 6,70 \\
\hline Promedio & 700 & 1,22 & 700 & 1,08 & 700 & 1,28 & 700 & 1,06 & 700 & 1,07 \\
\hline
\end{tabular}

Fuente: Organización de las Naciones Unidas para la Educación, la Ciencia y la Cultura (UNESCO), Informe de resultados TERCE (Tercer Estudio Regional Comparativo y Explicativo). Logros de aprendizaje, Santiago, 2016.

Por otra parte, cuando se analizan las diferencias promedio por grupos poblacionales: tipo de escuela (rural y urbana-pública), se observa una superioridad en los resultados de niños y niñas que asisten a las escuelas urbanas y de gestión pública en todos los países, sin distinción del grado ni tipo de prueba. Esta superioridad se evidencia más en aquellos países con mayor porcentaje de ruralidad (Guatemala, Honduras y Nicaragua) comparados con los de menor porcentaje (Argentina, Chile y Uruguay) (véanse los cuadros A1.4 y A1.5). 
La muestra de estudiantes seleccionados para la prueba TERCE muestra características sociodemográficas altamente heterogéneas. Hay una presencia ligeramente superior de niñas en los dos grados a nivel de país y de estudiantes provenientes de planteles educativos urbanos (a diferencia de países centroamericanos). Con excepción de Chile, más del $65 \%$ de los estudiantes, tanto en tercer grado como sexto grado, tienen madres o padres con bajos niveles educativos (escolaridad inferior a la de la Clasificación Internacional Normalizada de la Educación (CINE) P3)4 (véase el cuadro A1.5).

Una alternativa para medir la magnitud del efecto de asistir a un establecimiento de educación inicial en el TERCE es la medida "d" de Cohen (1988, 1992), que expresa la diferencia de media entre dos grupos en unidades de desvío estándar. Para el autor, valores de $\mathrm{d}=0,8$, implican un efecto grande, $d=0,50$ efecto moderado y $d=0,20$ efecto pequeño. Lo ideal es comparar dicha medida con otros efectos encontrados en la literatura a partir de estudios de un fenómeno similar. Así, Wen y otros (2012), al comparar diferencias en el resultado de Matemáticas y Lenguaje, por asistir un año o dos años a la educación preescolar, encuentran valores de "d" en un rango de [0,27-0,96], clasificándolos como de tamaño moderado y grandes. En un estudio reciente, Shah y otros (2017), observan el impacto de la educación preescolar en los rendimientos en matemáticas, vocabulario y habilidades ejecutivas, en primer y segundo grado, encontrando valores de "d" $=[0,22-0,40]$.

Como puede observarse en el cuadro 3, los efectos encontrados (muestra original) podrían clasificarse en pequeños y moderados, pues los valores de "d" para los países y las distintas pruebas se encuentran en un rango de 0,32 $<d \leqq 0,75$ (véanse en el cuadro A1.3 los valores al aplicar el emparejamiento por puntaje de propensión).

Cuadro 3

Tamaño del efecto "d" de Cohen, muestra original

\begin{tabular}{|c|c|c|c|c|c|}
\hline \multirow{2}{*}{ País } & \multicolumn{2}{|c|}{ Matemáticas } & \multicolumn{2}{|c|}{ Lectura } & \multirow{2}{*}{$\begin{array}{c}\text { Ciencias } \\
\text { Sexto grado }\end{array}$} \\
\hline & Tercer grado & Sexto grado & Tercer grado & Sexto grado & \\
\hline Argentina & 0,41 & 0,38 & 0,41 & 0,40 & 0,32 \\
\hline Brasil & 0,48 & 0,56 & 0,45 & 0,64 & 0,50 \\
\hline Chile & 0,43 & 0,38 & 0,40 & 0,33 & 0,34 \\
\hline Colombia & 0,67 & 0,66 & 0,69 & 0,69 & 0,52 \\
\hline Costa Rica & 0,46 & 0,51 & 0,49 & 0,50 & 0,45 \\
\hline Ecuador & 0,34 & 0,38 & 0,41 & 0,51 & 0,41 \\
\hline Guatemala & 0,75 & 0,57 & 0,74 & 0,61 & 0,60 \\
\hline Honduras & 0,77 & 0,66 & 0,75 & 0,73 & 0,66 \\
\hline México & 0,53 & 0,45 & 0,53 & 0,51 & 0,45 \\
\hline Nicaragua & 0,53 & 0,51 & 0,53 & 0,53 & 0,53 \\
\hline Paraguay & 0,60 & 0,64 & 0,73 & 0,74 & 0,68 \\
\hline Perú & 0,58 & 0,62 & 0,58 & 0,67 & 0,54 \\
\hline República Dominicana & 0,54 & 0,42 & 0,52 & 0,47 & 0,45 \\
\hline Uruguay & 0,58 & 0,57 & 0,50 & 0,53 & 0,55 \\
\hline
\end{tabular}

Fuente: Elaboración propia.

Nota: De acuerdo al tamaño del coeficiente "d", se puede clasificar el efecto en: $d \leqq 0,20$ muy pequeño, $0,20<d \leqq 0,50$ tamaño pequeño, 0,50 $<d \leqq 0,80$ tamaño moderado, $d>0,80$ tamaño grande (muestra original).

El menor efecto corresponde a la Argentina y en la prueba de Ciencias de sexto grado $(d=0,32)$ y el mayor efecto corresponde a la prueba de Lectura de tercer grado en Honduras $(d=0,75)$. Por otro parte, se puede observar un comportamiento dispar respecto del aumento o disminución del tamaño

${ }^{4}$ El nivel 3 de la Clasificación Internacional Normalizada de la Educación (CINE) de los programas educativos (CINE-P) corresponde a la educación secundaria superior. 
del efecto cuando se comparan el tercer y el sexto grado. Por ejemplo, en el caso de Matemáticas, países como la Argentina, Chile, Colombia y el Uruguay el tamaño del efecto disminuye, mientras que para el Brasil, Costa Rica y el Perú el tamaño se ha incrementado. Sin embargo, en todos los países y para todas las pruebas el tamaño clasificado como pequeño en tercero siguió siendo el mismo en sexto y aquellos que resultaron de tamaño moderado tampoco se modificaron.

\section{Resultados}

Antes de presentar los resultados obtenidos con el control sintético que genera el emparejamiento por puntaje de propensión, se estimó el efecto utilizando un modelo de mínimos cuadrados ordinarios con controles de individuo, familia, colegio y ciudad para cada asignatura y para cada país. Los resultados muestran que no hay evidencia de un efecto significativo de asistir al preescolar en el rendimiento en las pruebas TERCE, salvo algunas excepciones (el cuadro 4). No obstante esto, la discusión mencionada previamente sobre la importancia de aislar el efecto de variables no observables, o por lo menos de considerar controles sintéticos, nos llevó a los resultados del emparejamiento por puntaje de propensión.

Cuadro 4

Resultados de mínimos cuadrados ordinarios

\begin{tabular}{|c|c|c|c|c|c|c|c|c|c|c|c|c|c|}
\hline & Argentina & Brasil & Uruguay & Paraguay & Colombia & México & Perú & Ecuador & Nicaragua & Guatemala & Honduras & $\begin{array}{c}\text { República } \\
\text { Dominicana }\end{array}$ & Chile \\
\hline \multicolumn{14}{|c|}{ Tercer grado } \\
\hline \multicolumn{14}{|l|}{ Lectura } \\
\hline \multirow[t]{2}{*}{ Preescolar } & $0,181^{\star \star}$ & 0,0878 & 0,0699 & 0,146 & 0,175 & 0,0264 & $0,0961^{* *}$ & $0,0995^{\star \star}$ & 0,104 & $0,122^{\star \star}$ & 0,154 & 0,0882 & 0,0390 \\
\hline & $(0,0708)$ & 0 & $(0,0953)$ & 0 & 0 & 0 & $(0,0417)$ & $(0,0497)$ & $(0,0643)$ & $(0,0553)$ & 0 & $(0,0690)$ & 0 \\
\hline$N$ & 917 & 1339 & 994 & 1342 & 1666 & 1922 & 2362 & 1755 & 1384 & 1931 & 1624 & 1092 & 2224 \\
\hline $\begin{array}{l}\text { Cuadrado } \\
\text { ordinario }\end{array}$ & 0,233 & 0,261 & 0,350 & 0,198 & 0,342 & 0,270 & 0,255 & 0,285 & 0,209 & 0,313 & 0,211 & 0,165 & 0,184 \\
\hline \multicolumn{14}{|c|}{ Matemáticas } \\
\hline \multirow[t]{2}{*}{ Preescolar } & $0,173^{\star \star}$ & 0,130 & 0,0577 & 0,204 & 0,103 & 0,113 & 0,0654 & 0,0472 & $0,101^{*}$ & $0,0932^{*}$ & 0,0874 & 0,0784 & 0,0386 \\
\hline & $(0,0769)$ & 0 & $(0,0823)$ & 0 & 0 & 0 & $(0,0465)$ & $(0,0466)$ & $(0,0560)$ & $(0,0502)$ & 0 & $(0,0615)$ & 0 \\
\hline N & 914 & 1337 & 995 & 1324 & 1657 & 1910 & 2342 & 1735 & 1369 & 1907 & 1615 & 1091 & 2217 \\
\hline $\begin{array}{l}\text { Cuadrado } \\
\text { ordinario }\end{array}$ & 0,199 & 0,287 & 0,316 & 0,147 & 0,349 & 0,258 & 0,243 & 0,178 & 0,187 & 0,334 & 0,176 & 0,165 & 0,192 \\
\hline \multicolumn{14}{|c|}{ Sexto grado } \\
\hline \multicolumn{14}{|l|}{ Lectura } \\
\hline \multirow[t]{2}{*}{ Preescolar } & 0,0298 & $0,277^{\star \star \star}$ & 0,177 & $0,142^{\star *}$ & 0,172 & 0,131 & $0,196^{\star \star \star}$ & 0,174 & $0,151^{\star \star \star}$ & 0,0721 & 0,0904 & $0,101^{\star}$ & $0,0985^{\star \star}$ \\
\hline & $(0,0479)$ & $(0,0812)$ & 0 & $(0,0554)$ & 0 & 0 & $(0,0373)$ & 0 & $(0,0474)$ & $(0,0492)$ & 0 & $(0,0561)$ & $(0,0426)$ \\
\hline N & 1626 & 1224 & 1304 & 1592 & 2189 & 2331 & 2753 & 2225 & 1820 & 2350 & 2169 & 1706 & 2695 \\
\hline $\begin{array}{l}\text { Cuadrado } \\
\text { ordinario }\end{array}$ & 0,173 & 0,211 & 0,199 & 0,322 & 0,176 & 0,217 & 0,320 & 0,245 & 0,190 & 0,260 & 0,220 & 0,177 & 0,143 \\
\hline \multicolumn{14}{|c|}{ Matemáticas } \\
\hline \multirow[t]{2}{*}{ Preescolar } & 0,0792 & $0,169^{\star \star}$ & 0,151 & $0,160^{\star \star \star}$ & 0,171 & 0,166 & $0,244^{\star \star \star}$ & 0,0752 & $0,133^{\star \star \star}$ & $0,114^{\star \star}$ & 0,147 & $0,151^{\star \star \star}$ & $0,155^{\star \star \star}$ \\
\hline & $(0,0555)$ & $(0,0728)$ & 0 & $(0,0522)$ & 0 & 0 & $(0,0434)$ & 0 & $(0,0409)$ & $(0,0477)$ & 0 & $(0,0475)$ & $(0,0452)$ \\
\hline N & 1521 & 1207 & 1259 & 1538 & 2108 & 2289 & 2733 & 2181 & 1739 & 2283 & 2089 & 1539 & 2649 \\
\hline $\begin{array}{l}\text { Cuadrado } \\
\text { ordinario }\end{array}$ & 0,132 & 0,178 & 0,213 & 0,171 & 0,144 & 0,168 & 0,263 & 0,169 & 0,129 & 0,215 & 0,111 & 0,089 & 0,113 \\
\hline
\end{tabular}

Fuente: Elaboración propia.

Nota: Errores estándar entre paréntesis: ${ }^{* \star} p<0,01 ;{ }^{* \star} p<0,05,{ }^{*} p<0,1$.

a Todas las regresiones incluyen controles de estudiante, de familia y de colegio. 
Para claridad de los resultados de emparejamiento por puntaje de propensión, se presentan los coeficientes estimados de las variables estandarizadas, luego de centralizar las variables con media 0 y desviación estándar $=1^{5}$. Ello permite comprender mejor los efectos dado que las heterogeneidades regionales en términos de desarrollo hacen que ante un mismo cambio bruto en una asignatura tenga efectos relativos muy diferentes.

El cálculo del efecto promedio del tratamiento sobre el tratado incluyó diferentes especificaciones de acuerdo a diferentes controles. La decisión se fundamentó en los valores de B y R propuestos por Rubin (2001) en las distintas formas de emparejamiento (Nearest Neighbor 1:1, Caliper 1:1 con reemplazo y Kernel) sin observar en un inicio el valor del efecto promedio del tratamiento sobre el tratado. De esta forma, se seleccionó la estrategia de Kernel, con las covariables descritas anteriormente. Por otra parte, se observó que algunas variables no lograban un balance adecuado en algunos países (por ejemplo, género), por lo que se probó una especificación sin esta variable y los resultados no cambiaron sustancialmente.

Los resultados del efecto promedio del tratamiento sobre el tratado para todos los países para las pruebas evaluadas en tercer grado y sexto grado se pueden observar en el cuadro 5. En este punto, se siguió la metodología propuesta por DuGoff, Schuler y Stuart (2014) para incorporar los pesos muestrales en la estimación del efecto promedio del tratamiento sobre el tratado mediante el emparejamiento por puntaje de propensión. Finalmente, para efectos de claridad y de robustez de los resultados, el emparejamiento por puntaje de propensión se llevó a cabo eliminando una covariable (genero del estudiante). El objetivo fue observar grandes modificaciones en las estimaciones realizadas y se pudo identificar la estabilidad de los cálculos. Se ejecutó el análisis de sensibilidad propuesto por Rosenbaum (2002). Los valores de los cocientes de probabilidades (odds ratios, gamma) a partir de los cuales las estimaciones son sensibles a sesgo oculto se encuentran en un rango de $[1,05 ; 1,65]^{6}$. La mayoría de los países fueron sensibles a partir de valores gamma de 1,20 (nivel de confianza 95\%)

En el corto plazo (tercer grado) se encuentra para la mayoría de los países un efecto positivo tanto en Matemáticas como en Lectura, pero el tamaño del efecto sí tiene algunas diferencias notorias. En Matemáticas, para países como Honduras y el Paraguay, el aporte de preescolar fluctúa entre el 0,25 y el 0,33 de una desviación estándar. El tamaño, en términos de puntaje, equivale a un rango de 24 y 31 puntos en la prueba (efecto promedio del tratamiento sobre el tratado de la muestra), pero los efectos se igualan al analizar el efecto promedio del tratamiento sobre el tratado de la población objetivo, dejando de ser significativo para Honduras. Entre los demás países, en general el efecto estimado (efecto promedio del tratamiento sobre el tratado y efecto promedio del tratamiento sobre el tratado de la población objetivo) oscila entre 0,08 y 0,2 desviaciones estándar, lo que se encuentra dentro de los valores esperados de acuerdo a otras estimaciones de la literatura mencionada previamente. En el caso del área de Lectura, el efecto es de menor tamaño en la gran mayoría de países, con excepción del Ecuador, Guatemala y la República Dominicana (efecto promedio del tratamiento sobre el tratado de la muestra), agregándose Colombia y el Uruguay al analizar el efecto promedio del tratamiento sobre el tratado de la población objetivo. Esta característica parecería sugerir que las actividades realizadas en Matemáticas en el sistema educativo generan una mayor ventaja que las relacionadas con Lenguaje. No es extraño este aspecto, si se tiene en cuenta que las personas empiezan a manejar el lenguaje desde edades muy tempranas en sus propios hogares.

\footnotetext{
5 En el cuadro A1.2 se presentan los coeficientes de la estimación por emparejamiento por puntaje de propensión (efecto promedio del tratamiento sobre el tratado de la muestra).

6 Es difícil de explicar la existencia de problemas en la variabilidad de los datos para Panamá desde la creación de la base y por ello optamos por no incluirla en el estudio.

7 Debido a la extensión de los cálculos, no se incluyen en el documento pero están disponibles a solicitud.
} 


\section{Cuadro 5}

Resultados del emparejamiento por puntaje de propensión: efecto promedio del tratamiento sobre el tratado en la población

\begin{tabular}{|c|c|c|c|c|c|c|c|c|}
\hline & \multicolumn{4}{|c|}{ Tercer grado } & \multicolumn{4}{|c|}{ Sexto grado } \\
\hline & $\begin{array}{l}\text { Efecto promedio del } \\
\text { tratamiento sobre } \\
\text { el tratado de la } \\
\text { población objetivo }\end{array}$ & $\begin{array}{c}\text { Media } \\
\text { (sesgo) }\end{array}$ & $\begin{array}{l}\text { Balance con } \\
\text { el puntaje de } \\
\text { propensión } \\
\text { (B) } \\
\end{array}$ & $\begin{array}{l}\text { Razón de } \\
\text { varianza } \\
\text { (R) }\end{array}$ & $\begin{array}{l}\text { Efecto promedio del } \\
\text { tratamiento sobre } \\
\text { el tratado de la } \\
\text { población objetivo }\end{array}$ & $\begin{array}{c}\text { Media } \\
\text { (sesgo) }\end{array}$ & $\begin{array}{l}\text { Balance con } \\
\text { puntaje de } \\
\text { propensión } \\
\text { (B) }\end{array}$ & $\begin{array}{l}\text { Razón de } \\
\text { varianza } \\
\text { (R) }\end{array}$ \\
\hline \multicolumn{9}{|l|}{ Argentina } \\
\hline Matemáticas & $0,191^{\star *}$ & 1,1 & 6,8 & 0,99 & $0,127^{\star \star}$ & 3,1 & 12,9 & 1 \\
\hline Lectura & $0,112^{\star *}$ & 1,1 & 6,8 & 0,99 & $0,07 \sim$ & 3,1 & 12,9 & 1 \\
\hline Ciencia & & & & & $0,09 \sim$ & 3,1 & 12,9 & 1 \\
\hline \multicolumn{9}{|l|}{ Brasil } \\
\hline Matemáticas & $0,142^{\star \star}$ & 2,4 & 9,2 & 1 & $0,210^{* *}$ & 1,7 & 8,5 & 1,16 \\
\hline Lectura & $0,07 \sim$ & 2,4 & 9,2 & 1 & $0,316^{\star *}$ & 1,7 & 8,5 & 1,16 \\
\hline Ciencia & & & & & $0,194^{\star *}$ & 1,7 & 8,5 & 1,16 \\
\hline \multicolumn{9}{|l|}{ Uruguay } \\
\hline Matemáticas & $0,06 \sim$ & 3 & 14 & 0,93 & $0,179^{\star *}$ & 1,9 & 8,6 & 1 \\
\hline Lectura & $0,09^{\star}$ & 3 & 14 & 0,93 & $0,152^{\star \star}$ & 1,9 & 8,6 & 1 \\
\hline Ciencia & & & & & $0,135^{\star \star}$ & 1,9 & 8,6 & 1 \\
\hline \multicolumn{9}{|l|}{ Paraguay } \\
\hline Matemáticas & $0,220^{\text {** }}$ & 1,4 & 5,7 & 1,33 & $0,139^{\star *}$ & 1,7 & 7,8 & 1,21 \\
\hline Lectura & $0,173^{\star *}$ & 1,4 & 5,7 & 1,33 & $0,03 \sim$ & 1,7 & 7,8 & 1,21 \\
\hline Ciencia & & & & & $0,120^{* *}$ & 1,7 & 7,8 & 1,21 \\
\hline \multicolumn{9}{|l|}{ Colombia } \\
\hline Matemáticas & $0,06 \sim$ & 2,1 & 12,4 & 0,91 & $0,170^{* *}$ & 2 & 8,5 & 1,16 \\
\hline Lectura & $0,154^{\star \star}$ & 2,1 & 12,4 & 0,91 & $0,07^{\star}$ & 2 & 8,5 & 1,16 \\
\hline Ciencia & & & & & $0,132^{\star *}$ & 2 & 8,5 & 1,16 \\
\hline \multicolumn{9}{|l|}{ México } \\
\hline Matemáticas & $0,087^{\star \star}$ & 2,7 & 14,7 & 1,09 & $0,142^{\star *}$ & 2,4 & 9 & 1,17 \\
\hline Lectura & $0,065 \sim$ & 2,7 & 14,7 & 1,09 & $0,09^{\star \star}$ & 2,4 & 9 & 1,17 \\
\hline Ciencia & & & & & $0,121^{\text {** }}$ & 2,4 & 9 & 1,17 \\
\hline \multicolumn{9}{|l|}{ Perú } \\
\hline Matemáticas & $0,085^{\star \star}$ & 1,5 & 7,9 & 1,02 & $0,207^{* *}$ & 1,2 & 5,2 & 0,99 \\
\hline Lectura & $0,090^{\star *}$ & 1,5 & 7,9 & 1,02 & $0,164^{\star *}$ & 1,2 & 5,2 & 0,99 \\
\hline Ciencia & & & & & $0,121^{\text {** }}$ & 1,2 & 5,2 & 0,99 \\
\hline \multicolumn{9}{|l|}{ Ecuador } \\
\hline Matemáticas & $0,129^{\star \star}$ & 2 & 9 & 1 & $0,05 \sim$ & 2 & 8,9 & 1,04 \\
\hline Lectura & $0,145^{\star \star}$ & 2 & 9 & 1 & $0,128^{\star *}$ & 2 & 8,9 & 1,04 \\
\hline Ciencia & & & & & $0,138^{\star *}$ & 2 & 8,9 & 1,04 \\
\hline \multicolumn{9}{|l|}{ Panamá } \\
\hline Matemáticas & $0,04 \sim$ & 1,5 & 7,3 & 0,91 & $0,02 \sim$ & 1,6 & 8,4 & 1 \\
\hline Lectura & $-0,06 \sim$ & 1,5 & 7,3 & 0,91 & $0,02 \sim$ & 1,6 & 8,4 & 1 \\
\hline Ciencia & & & & & $0,07 \sim$ & 1,6 & 8,4 & 1 \\
\hline
\end{tabular}


Cuadro 5 (conclusión)

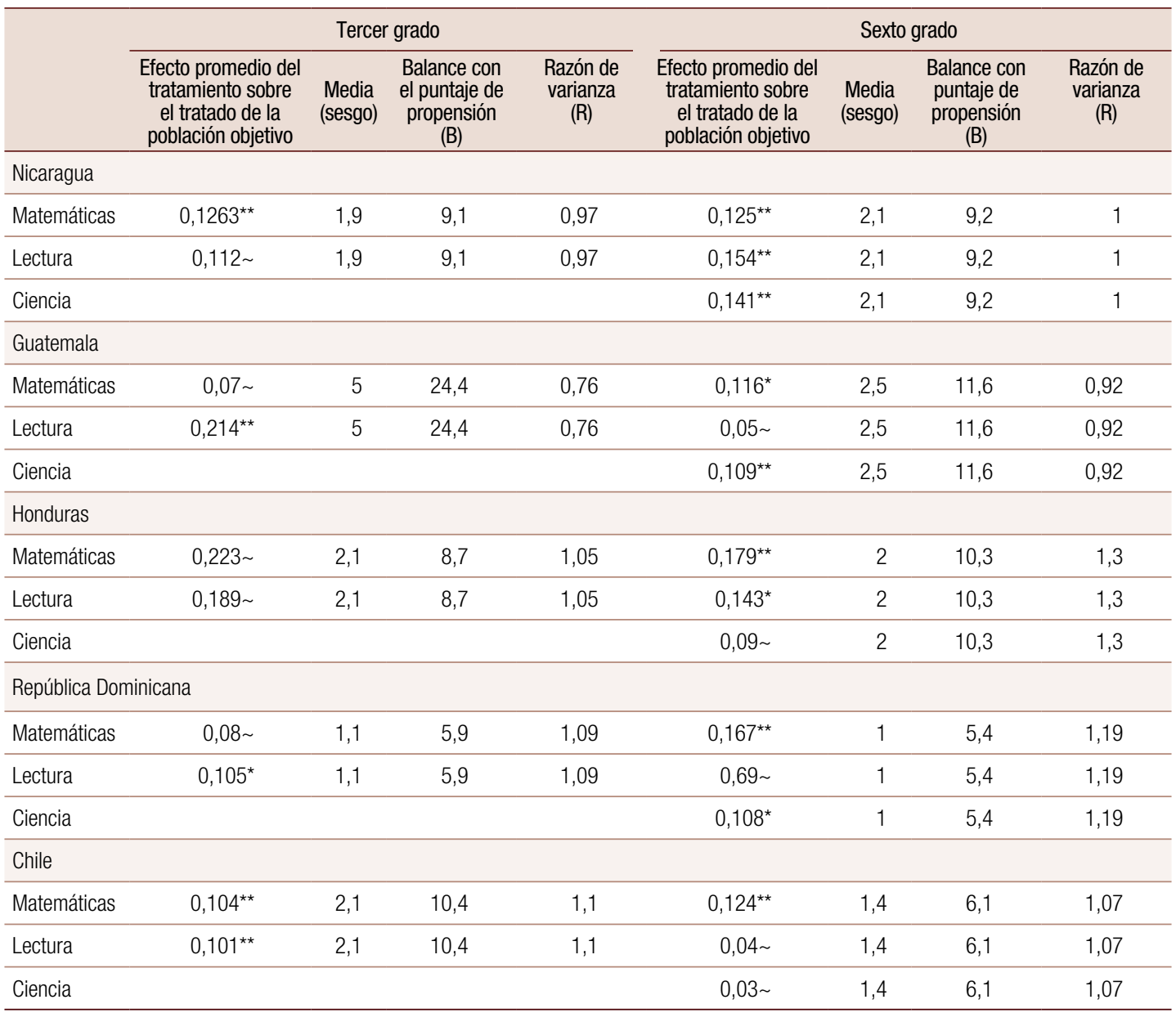

Fuente: Elaboración propia.

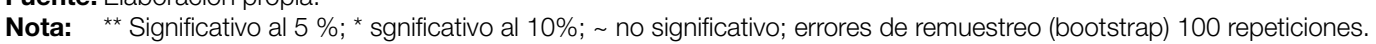

Cuando se observan los resultados para sexto grado, en que se evalúa también el área de Ciencias, se encuentran algunas particularidades. En primer lugar, en la gran mayoría de países se acentúa la importancia del preescolar en Matemáticas (efecto promedio del tratamiento sobre el tratado de la muestra y efecto promedio del tratamiento sobre el tratado de la población objetivo) y solo en la Argentina, el Ecuador, Honduras y el Paraguay decrece el tamaño del coeficiente del efecto promedio del tratamiento sobre el tratado de la población objetivo. En Lectura, parecería evidenciarse un resultado contrario en muchos países. Es así como en los países del Cono Sur (Argentina, Chile, Paraguay) disminuye el efecto de haber asistido a educación inicial sobre el puntaje de la prueba del TERCE (efecto promedio del tratamiento sobre el tratado de la muestra y efecto promedio del tratamiento sobre el tratado de la población objetivo) y en países altamente poblados como el Brasil, Colombia y México se da un incremento en el coeficiente de efecto causal. En segundo lugar, la magnitud de los cambios es heterogénea. Esto es, mientras que en el Brasil pasa de 0,07 (no significativo) en Lectura en tercer grado a 0,31 en sexto grado, en el Paraguay pasa de 0,173 en Lectura en tercer grado a 0,03 (no significativo) en sexto grado (efecto promedio del tratamiento sobre el tratado de la población objetivo). 
Independientemente de si existe un efecto de mediano plazo creciente o decreciente de asistir al preescolar en los resultados de la prueba, el Brasil, Nicaragua, el Paraguay y la República Dominicana muestran una variación notoria en estos coeficientes. En tercer lugar, aunque sigue siendo más grande el efecto sobre Matemáticas, el caso de Ciencias es importante. En muchos casos, podría decirse que la experimentación y el aprendizaje dirigido, aprendiendo a seguir instrucciones desde pequeño, puede estimular la adquisición de competencias científicas hacia las ciencias y de ahí que asistir al preescolar genere resultados que fluctúan entre 0,07 y 0,2 desviaciones estándar en el puntaje del TERCE para los alumnos de sexto grado (10 de los 14 países considerados tienen un coeficiente superior a 0,10 desviaciones estándar en el efecto promedio del tratamiento sobre el tratado de la población objetivo).

Los resultados anteriores ofrecen evidencia en favor de seguir trabajando por aumentar la cobertura de la educación preescolar, puesto que, además de los beneficios mencionados directamente para los estudiantes, existe evidencia de beneficios indirectos sobre la equidad, la participación laboral de las madres y las condiciones de salud.

\section{Conclusiones}

Actualmente prevalecen diferencias considerables en el acceso a educación preescolar en América Latina y dichas diferencias se acentúan entre los estudiantes de zonas urbanas y rurales.

Los resultados obtenidos al utilizar el emparejamiento por puntaje de propensión sobre la prueba TERCE parecen indicar que hay efectos positivos de asistir al preescolar y que no se extinguen aún seis años después de haber cursado el preescolar. Este resultado es importante en términos de política educativa porque muestra que es necesario reducir la inequidad de oportunidades al inicio del ciclo educativo, mediante inversiones necesarias para garantizar el ingreso de los niños al sistema educativo a una menor edad.

No obstante esto, los resultados son heterogéneos entre países, asignaturas y período para la prueba TERCE. Esto quiere decir que no se puede encontrar a partir de esta evidencia una tendencia creciente o decreciente para el conjunto de países estudiados. La existencia de políticas internas podrá ayudar a reducir esta inequidades en el acceso y así potencializar los resultados cognitivos y no cognitivos de los más vulnerables. Si bien hay aspectos institucionales muy diversos entre ellos, lo que se puede afirmar es que, en promedio, la tendencia general en Matemáticas es distinta a la de Lectura. Para el área de Ciencias, también se encuentra un impacto positivo de la asistencia al preescolar y el tamaño encontrado del efecto no es insignificante luego de hacer los controles sintéticos mencionados.

Los resultados mencionados permiten generar una evidencia importante para continuar con los esfuerzos por lograr una cobertura universal, que amplíen las posibilidades de desarrollo, al proveer elementos complementarios para la educación de los niños y ampliar las posibilidades de participación laboral de las madres. No se debe olvidar que, al recibir estudiantes a la menor edad posible, se incrementan las posibilidades de tener mayores logros cognitivos y no cognitivos. Estos últimos son muy importantes para que comunidades vulnerables puedan alcanzar otros objetivos en materia de salud y nutrición.

Dada la evidencia resultante, es necesario insistir en que se hagan mayores esfuerzos por parte de los Gobiernos respecto de la educación inicial, lo que debe ser una tarea continua, con asignación de los recursos necesarios, no solo para expandir su cobertura, sino para asegurar la mejora de la calidad de dichos programas. Dada la rentabilidad de este tipo de inversiones, es importante monitorear tanto calidad como cobertura en zonas rurales para que la situación de inequidad en el acceso en América Latina disminuya. Solo así podrá decirse que la educación preescolar está garantizando los derechos del niño. 


\section{Bibliografía}

Abadie, A. y G. Imbens (2005), "On the failure of the bootstrap for matching estimators" [en línea] http:// www.princeton.edu/ erp/Econometrics/Old\%20Pdfs/Abadie.pdf.

Araujo, M., M. Dormal y N. Schady (2017), "La calidad de los jardines de cuidado infantil y el desarrollo infantil", Documento de Trabajo del BID, № 779, Washington, D.C., Banco Interamericano de Desarrollo (BID).

Behrman, J., Y. Cheng y P. Todd (2004), "Evaluating preschool programs when length of exposure to the program varies: a nonparametric approach", The Review of Economics and Statistics, vol. 86, № 1 , Cambridge, MIT Press.

Berlinski, S., S. Galiani y M. Manacorda (2008), "Giving children a better start: preschool attendance and school-age profiles", Journal of Public Economics, vol. 92, № 5-6, Amsterdam, Elsevier.

Berlinski, S. y N. Schady (eds.) (2015), Los primeros años: el bienestar infantil y el papel de las políticas públicas, Washington, D.C., Banco Interamericano de Desarrollo (BID).

Bernal, R. y A. Camacho (2012), "La política de primera infancia en el contexto de la equidad y movilidad social en Colombia", Documentos CEDE, Nㅜ 33, Bogotá, Universidad de los Andes.

Bernal, R. y otros (2015), "The effects of the transition from home-based childcare to center-based childcare in Colombia", Bogotá, Universidad de los Andes, inédito. (2012), Evaluación de impacto del Programa Jardines Sociales/Centros de Desarrollo Infantil. Informe final de impacto, Bogotá, Universidad de los Andes.

Black, D. (2015), "Matching as a regression estimator", IZA World of Labor, № 186, Bonn.

Brookhart, M. y otros (2006), "Variable selection for propensity score models", American Journal of Epidemiology, vol. 163, No 12, Oxford, Oxford University Press.

Caliendo, M. y S. Kopeinig (2008), "Some practical guidance for the implementation of propensity score matching", Journal of Economic Surveys, vol. 22, № 1, Hoboken, Wiley.

Cascio, E. (2015), "The promises and pitfalls of universal early education", IZA World of Labor, N 116, Bonn.

Cohen, J. (1992), "A power primer", Psychological Bulletin, vol. 112, № 1, Washington, D.C., American Psychological Association.

(1988), Statistical Power Analysis for the Behavioral Sciences, Hillsdale, Lawrence Erlbaum Associates.

DuGoff, E., M. Schuler y E. Stuart (2014), "Generalizing observational study results: applying propensity score methods to complex surveys", Health Services Research, vol. 49, № 1, Hoboken, Wiley.

Gamboa, L. y N. Krüger (2016), "¿Existen diferencias en América Latina en el aporte de la educación preescolar al logro educativo futuro?: PISA 2009-2012", Revista CEPAL, № 118 (LC/G-2676-P), Santiago, Comisión Económica para América Latina y el Caribe (CEPAL).

Heckman, J., R. Pinto y P. Savelyev (2013), "Understanding the mechanisms through which an influential early childhood program boosted adult outcomes", American Economic Review, vol. 103, № 6, Nashville, American Economic Association.

Heckman, J. y R. Robb (1986), "Alternative methods for solving the problem of selection bias in evaluating the impact of treatments on outcomes", Drawing Inferences from Self-Selected Samples, H. Wainer (ed.), Nueva York, Springer.

Loeb, S. y otros (2007), "How much is too much? The influence of preschool centers on children's social and cognitive development", Economics of Education Review, vol. 26, № 1, Amsterdam, Elsevier.

Magnuson, K., C. Ruhm y J. Waldfogel (2007a), "Does prekindergarten improve school preparation and performance?", Economics of Education Review, vol. 26, № 1, Amsterdam, Elsevier. (2007b), "The persistence of preschool effects: do subsequent classroom experience matter?", Early Childhood Research Quarterly, vol. 22, № 1, Amsterdam, Elsevier.

Mateo, M. y L. Rodríguez-Chamussy (2013), "Childcare and women's labor participation: evidence for Latin America and the Caribbean”, IDB Technical Note, № 586, Washington, D.C., Banco Interamericano de Desarrollo (BID).

MINEDUC (Ministerio de Educación de Chile) (2014), "Estado del arte de la educación parvularia en Chile", Santiago.

Nores, M. y W. Barnett (2010), "Benefits of early childhood interventions across the world: (under) investing in the very young", Economics of Education Review, vol. 29, № 2, Amsterdam, Elsevier.

Rosenbaum, P. (2002), Observational Studies, Nueva York, Springer.

Rosenbaum, P. y D. Rubin (1983), "The central role of the propensity score in observational studies for causal effects”, Biometrika, vol. 70, № 1, Oxford, Oxford University Press. 
Rubin, D. (2001), "Using propensity scores to help design observational studies: application to the tobacco litigation", Health Services and Outcomes Research Methodology, vol. 2, № 3-4, Nueva York, Springer.

Schady, N. y otros (2015), "Wealth gradients in early childhood cognitive development in five Latin American countries", Journal of Human Resources, vol. 50, N² 2, Madison, University of Wisconsin Press.

Shah, H. y otros (2017), "One or two years of participation: is dosage of an enhanced publicly funded preschool program associated with the academic and executive function skills of low-income children in early elementary school?", Early Childhood Research Quarterly, vol. 40, Amsterdam, Elsevier.

Shonkoff, J. y D. Phillips (2000), From Neurons to Neighborhoods: The Science of Early Childhood Development, Washington, D.C., National Academy Press.

Skibbe, L. y otros (2011), "Schooling effects on preschoolers' self-regulation, early literacy, and language growth", Early Childhood Research Quarterly, vol. 26, № 1, Amsterdam, Elsevier.

SOPLA (Programa Regional sobre Políticas Sociales) (2014), Los desafíos de educación preescolar, básica y media en América Latina, Santiago, Konrad Adenauer Stiftung/Programa Regional sobre Políticas Sociales (SOPLA).

Stuart, E. (2010), "Matching methods for causal inference: a review and look forward", Statistical Science, vol. 25, N ${ }^{\circ}$ 1, Beachwood, Institute of Mathematical Statistics (IMS).

Temple, J. y A. Reynolds (2007), "Benefits and costs of investments in preschool education: evidence from the Child-Parent Centers and related programs", Economics of Education Review, vol. 26, № 1 , Amsterdam, Elsevier.

UNESCO (Organización de las Naciones Unidas para la Educación, la Ciencia y la Cultura) (2016), Reporte técnico. Tercer Estudio Regional Comparativo y Explicativo (TERCE), Santiago. (2014), Informe de Seguimiento de la EPT en el Mundo 2013/4. Enseñanza y aprendizaje: lograr la calidad para todos, París.

- (2013), Situación educativa de América Latina y el Caribe: hacia una educación para todos 2015, Santiago. (2008), Indicadores de la educación de la primera infancia en América Latina: propuesta y experiencias piloto, Santiago.

Wagstaff, A. (2007), "Health insurance for the poor: initial impacts of Vietnam's Health Care Fund for the Poor", Impact Evaluation Series, № 11, Washington, D.C., Banco Mundial.

Wen, X. y otros (2012), "Are two years better than one year? A propensity score analysis of the impact of Head Start program duration on children's school performance in kindergarten", Early Childhood Research Quarterly, vol. 27, № 4, Amsterdam, Elsevier. 


\section{Anexo A1}

Cuadro A1.1

Muestra efectiva del Tercer Estudio Regional Comparativo y Explicativo (TERCE)

\begin{tabular}{|c|c|c|c|c|c|c|}
\hline & \multicolumn{2}{|c|}{ Lectura } & \multicolumn{2}{|c|}{ Matemáticas } & \multicolumn{2}{|c|}{ Ciencias } \\
\hline & Tercer grado & Sexto grado & Tercer grado & Sexto grado & Tercer grado & Sexto grado \\
\hline Argentina & 3655 & 3658 & 3751 & 3639 & 3663 & 3632 \\
\hline Brasil & 3254 & 2900 & 3343 & 2983 & 2986 & 3908 \\
\hline Chile & 4751 & 5056 & 4709 & 5044 & 5029 & 4754 \\
\hline Colombia & 4018 & 4343 & 3975 & 4308 & 4325 & 4028 \\
\hline Costa Rica & 3427 & 3490 & 3428 & 3520 & 3520 & 3436 \\
\hline Ecuador & 4631 & 4842 & 4642 & 4818 & 4820 & 4621 \\
\hline Guatemala & 4060 & 3891 & 4282 & 4056 & 4070 & 4112 \\
\hline Honduras & 3743 & 3788 & 3870 & 3880 & 3886 & 3651 \\
\hline México & 3465 & 3554 & 3543 & 3618 & 3622 & 3456 \\
\hline Nicaragua & 3513 & 3470 & 3810 & 3726 & 3741 & 3537 \\
\hline Paraguay & 3123 & 3175 & 3271 & 3222 & 3231 & 3274 \\
\hline Perú & 4946 & 4739 & 5038 & 4789 & 4801 & 5003 \\
\hline República Dominicana & 3504 & 3588 & 3757 & 3661 & 3669 & 3652 \\
\hline Uruguay & 2663 & 2799 & 2728 & 2799 & 2803 & 2672 \\
\hline Total & 56036 & 56779 & 57561 & 57476 & 54055 & 56500 \\
\hline
\end{tabular}

Fuente: Organización de las Naciones Unidas para la Educación, la Ciencia y la Cultura (UNESCO), Informe de resultados TERCE (Tercer Estudio Regional Comparativo y Explicativo). Logros de aprendizaje, Santiago, 2016.

\section{Cuadro A1.2}

Resultados del emparejamiento por puntaje de propensión: efecto promedio del tratamiento sobre el tratado en la muestra

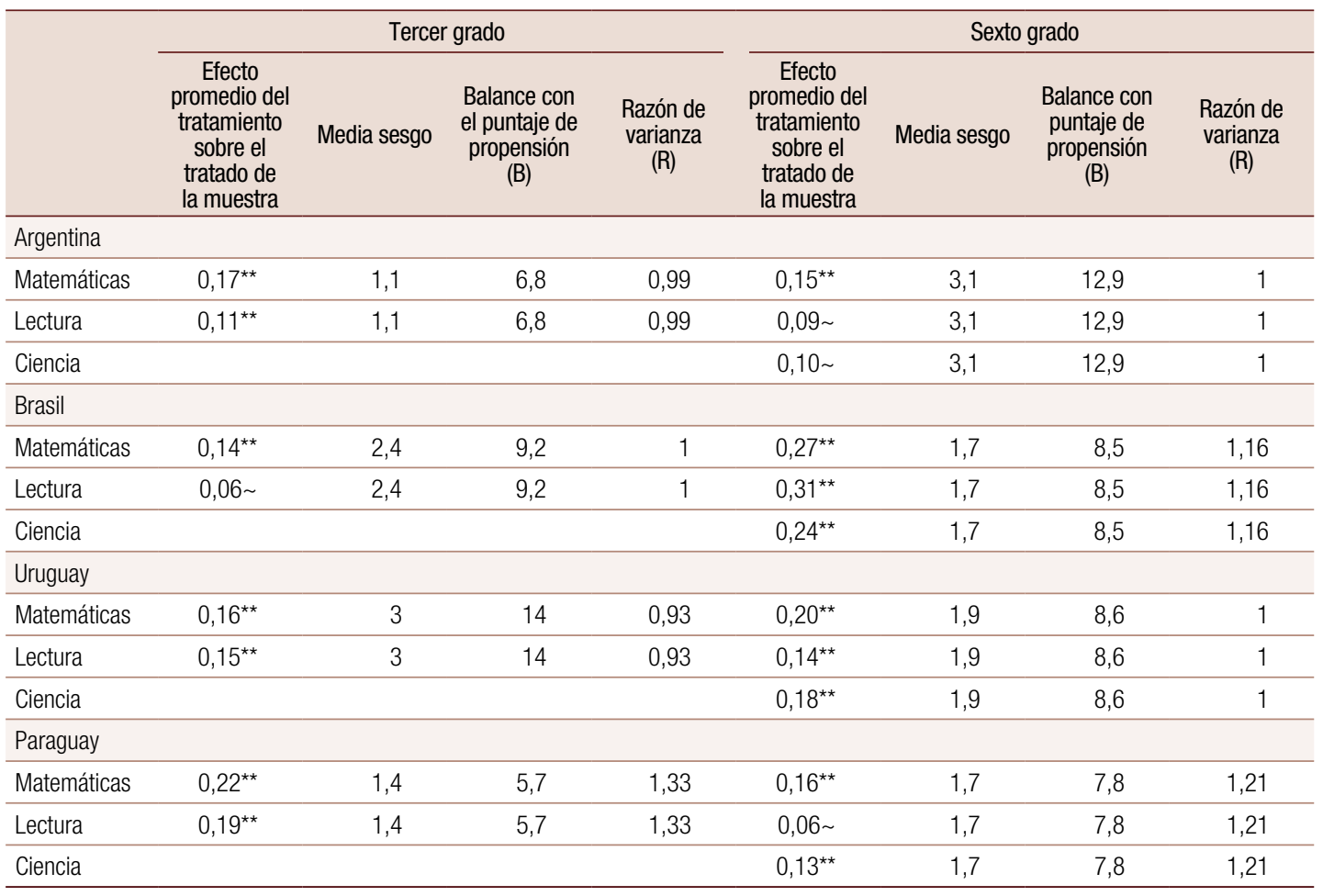


Cuadro A1.2 (conclusión)

\begin{tabular}{|c|c|c|c|c|c|c|c|c|}
\hline & \multicolumn{4}{|c|}{ Tercer grado } & \multicolumn{4}{|c|}{ Sexto grado } \\
\hline & $\begin{array}{c}\text { Efecto } \\
\text { promedio del } \\
\text { tratamiento } \\
\text { sobre el } \\
\text { tratado de } \\
\text { la muestra } \\
\end{array}$ & Media sesgo & $\begin{array}{l}\text { Balance con } \\
\text { el puntaje de } \\
\text { propensión } \\
\text { (B) }\end{array}$ & $\begin{array}{l}\text { Razón de } \\
\text { varianza } \\
\text { (R) }\end{array}$ & $\begin{array}{c}\text { Efecto } \\
\text { promedio del } \\
\text { tratamiento } \\
\text { sobre el } \\
\text { tratado de } \\
\text { la muestra } \\
\end{array}$ & Media sesgo & $\begin{array}{l}\text { Balance con } \\
\text { puntaje de } \\
\text { propensión } \\
\text { (B) }\end{array}$ & $\begin{array}{c}\text { Razón de } \\
\text { varianza } \\
\text { (R) }\end{array}$ \\
\hline \multicolumn{9}{|l|}{ Colombia } \\
\hline Matemáticas & $0,12^{\star \star}$ & 2,1 & 12,4 & 0,91 & $0,19^{\star \star}$ & 2 & 8,5 & 1,16 \\
\hline Lectura & $0,10^{\star \star}$ & 2,1 & 12,4 & 0,91 & $0,13^{\star \star}$ & 2 & 8,5 & 1,16 \\
\hline Ciencia & & & & & $0,09^{\star *}$ & 2 & 8,5 & 1,16 \\
\hline \multicolumn{9}{|l|}{ México } \\
\hline Matemáticas & $0,10^{\star \star}$ & 2,7 & 14,7 & 1,09 & $0,15^{\star \star}$ & 2,4 & 9 & 1,17 \\
\hline Lectura & $0,09 \sim$ & 2,7 & 14,7 & 1,09 & $0,10^{\star \star}$ & 2,4 & 9 & 1,17 \\
\hline Ciencia & & & & & $0,14^{\star \star}$ & 2,4 & 9 & 1,17 \\
\hline \multicolumn{9}{|l|}{ Perú } \\
\hline Matemáticas & $0,13^{\star \star}$ & 1,5 & 7,9 & 1,02 & $0,21^{\star \star}$ & 1,2 & 5,2 & 0,99 \\
\hline Lectura & $0,13^{\star \star}$ & 1,5 & 7,9 & 1,02 & $0,15^{\star \star}$ & 1,2 & 5,2 & 0,99 \\
\hline Ciencia & & & & & $0,11^{\star *}$ & 1,2 & 5,2 & 0,99 \\
\hline \multicolumn{9}{|l|}{ Ecuador } \\
\hline Matemáticas & $0,12^{\star \star}$ & 2 & 9 & 1 & $0,06 \sim$ & 2 & 8,9 & 1,04 \\
\hline Lectura & $0,14^{\star *}$ & 2 & 9 & 1 & $0,11^{* *}$ & 2 & 8,9 & 1,04 \\
\hline Ciencia & & & & & $0,11 \sim$ & 2 & 8,9 & 1,04 \\
\hline \multicolumn{9}{|l|}{ Nicaragua } \\
\hline Matemáticas & $0,16^{\star \star}$ & 1,9 & 9,1 & 0,97 & $0,19^{\star \star}$ & 2,1 & 9,2 & 1 \\
\hline Lectura & $0,08 \sim$ & 1,9 & 9,1 & 0,97 & $0,17^{\star \star}$ & 2,1 & 9,2 & 1 \\
\hline Ciencia & & & & & $0,22^{\star \star}$ & 2,1 & 9,2 & 1 \\
\hline \multicolumn{9}{|l|}{ Guatemala } \\
\hline Matemáticas & $0,08 \sim$ & 5 & 24,4 & 0,76 & $0,14^{\star \star}$ & 2,5 & 11,6 & 0,92 \\
\hline Lectura & $0,20^{\star \star}$ & 5 & 24,4 & 0,76 & $0,13^{\star \star}$ & 2,5 & 11,6 & 0,92 \\
\hline Ciencia & & & & & $0,15^{\star \star}$ & 2,5 & 11,6 & 0,92 \\
\hline \multicolumn{9}{|l|}{ Honduras } \\
\hline Matemáticas & $0,34^{\star \star}$ & 2,1 & 8,7 & 1,05 & $0,29^{\star \star}$ & 2 & 10,3 & 1,3 \\
\hline Lectura & $0,23^{\star \star}$ & 2,1 & 8,7 & 1,05 & $0,28^{\star \star}$ & 2 & 10,3 & 1,3 \\
\hline Ciencia & & & & & $0,29^{\star \star}$ & 2 & 10,3 & 1,3 \\
\hline \multicolumn{9}{|c|}{ República Dominicana } \\
\hline Matemáticas & $0,11 \sim$ & 1,1 & 5,9 & 1,09 & $0,18^{\star \star}$ & 1 & 5,4 & 1,19 \\
\hline Lectura & $0,20^{\star *}$ & 1,1 & 5,9 & 1,09 & $0,11 \sim$ & 1 & 5,4 & 1,19 \\
\hline Ciencia & & & & & $0,14^{\star \star}$ & 1 & 5,4 & 1,19 \\
\hline \multicolumn{9}{|l|}{ Chile } \\
\hline Matemáticas & $0,14^{\star \star}$ & 2,1 & 10,4 & 1,1 & $0,11^{\star \star}$ & 1,4 & 6,1 & 1,07 \\
\hline Lectura & $0,11^{\star \star}$ & 2,1 & 10,4 & 1,1 & $0,05 \sim$ & 1,4 & 6,1 & 1,07 \\
\hline Ciencia & & & & & $0,04 \sim$ & 1,4 & 6,1 & 1,07 \\
\hline
\end{tabular}

Fuente: Elaboración propia, sobre la base de datos del Tercer Estudio Regional Comparativo y Explicativo (TERCE).

Nota: $\quad$ ** Significancia al $5 \%$; * Significancia al 10\%; No significativo. 
Cuadro A1.3

Tamaño del efecto "d" de Cohen, muestra PS Asistió - No Asistió-

PS $^{*}=$ emparejamiento por puntaje de propensión

\begin{tabular}{|c|c|c|c|c|c|}
\hline \multirow{2}{*}{ País } & \multicolumn{2}{|c|}{ Matemáticas } & \multicolumn{2}{|c|}{ Lectura } & \multirow{2}{*}{$\begin{array}{c}\text { Ciencias } \\
\text { Sexto grado }\end{array}$} \\
\hline & Tercer grado & Sexto grado & Tercer grado & Sexto grado & \\
\hline Argentina & 0,39 & 0,43 & 0,42 & 0,41 & 0,30 \\
\hline Brasil & 0,43 & 0,57 & 0,40 & 0,70 & 0,57 \\
\hline Chile & 0,41 & 0,38 & 0,38 & 0,33 & 0,35 \\
\hline Colombia & 0,66 & 0,63 & 0,68 & 0,66 & 0,49 \\
\hline Ecuador & 0,33 & 0,38 & 0,39 & 0,52 & 0,42 \\
\hline Guatemala & 0,74 & 0,56 & 0,73 & 0,60 & 0,60 \\
\hline Honduras & 0,76 & 0,71 & 0,73 & 0,75 & 0,67 \\
\hline México & 0,48 & 0,43 & 0,47 & 0,47 & 0,44 \\
\hline Nicaragua & 0,46 & 0,51 & 0,46 & 0,55 & 0,53 \\
\hline Paraguay & 0,59 & 0,63 & 0,69 & 0,74 & 0,65 \\
\hline Perú & 0,55 & 0,61 & 0,55 & 0,65 & 0,52 \\
\hline República Dominicana & 0,48 & 0,41 & 0,55 & 0,43 & 0,43 \\
\hline Uruguay & 0,57 & 0,55 & 0,47 & 0,51 & 0,53 \\
\hline
\end{tabular}

Fuente: Elaboración propia, sobre la base de datos del Tercer Estudio Regional Comparativo y Explicativo (TERCE).

Nota: $\quad d \leqq 0,20$ muy pequeño $-0,20<d \leqq 0,50$ tamaño pequeño $-0,50<d \leqq 0,80$ tamaño moderado $d>0,80$ tamaño grande *Muestra después de aplicar el puntaje de propensión, soporte común.

\section{Cuadro A1.4}

Distribución de estudiantes por grupos socioeconómicos

\begin{tabular}{|c|c|c|c|c|c|c|c|c|c|c|c|c|c|c|c|}
\hline & ARG & BRA & CHL & COL & CRI & DOM & ECU & GTM & HND & MEX & NIC & PRY & PER & URY & Total \\
\hline \multicolumn{16}{|c|}{ Sexto grado } \\
\hline \multicolumn{16}{|c|}{ Edad del estudiante } \\
\hline 11 años & 0,15 & 0,00 & 0,06 & 0,12 & 0,00 & 0,20 & 0,32 & 0,02 & 0,07 & 0,02 & 0,14 & 0,12 & 0,28 & 0,03 & 0,11 \\
\hline 12 años & 0,68 & 0,27 & 0,76 & 0,49 & 0,52 & 0,43 & 0,54 & 0,23 & 0,50 & 0,72 & 0,46 & 0,62 & 0,51 & 0,77 & 0,55 \\
\hline 13 a15 años & 0,17 & 0,73 & 0,18 & 0,39 & 0,48 & 0,37 & 0,14 & 0,75 & 0,43 & 0,26 & 0,40 & 0,25 & 0,21 & 0,20 & 0,34 \\
\hline Niña & 0,49 & 0,52 & 0,51 & 0,50 & 0,50 & 0,51 & 0,47 & 0,49 & 0,50 & 0,50 & 0,54 & 0,51 & 0,50 & 0,52 & 0,50 \\
\hline Colegio rural & 0,34 & 0,18 & 0,22 & 0,38 & 0,09 & 0,35 & 0,27 & 0,66 & 0,55 & 0,27 & 0,51 & 0,39 & 0,31 & 0,14 & 0,34 \\
\hline $\begin{array}{l}\text { Colegio urbano- } \\
\text { público }\end{array}$ & 0,42 & 0,50 & 0,20 & 0,20 & 0,71 & 0,44 & 0,48 & 0,25 & 0,12 & 0,47 & 0,27 & 0,25 & 0,37 & 0,67 & 0,37 \\
\hline \multicolumn{16}{|c|}{ Nivel educativo de la madre } \\
\hline $\begin{array}{l}\text { No tiene } \\
\text { estudios }\end{array}$ & 0,03 & 0,15 & 0,03 & 0,03 & 0,06 & 0,07 & 0,05 & 0,18 & 0,13 & 0,07 & 0,09 & 0,03 & 0,07 & 0,01 & 0,07 \\
\hline [CINE-P 1-2] & 0,40 & 0,31 & 0,21 & 0,29 & 0,42 & 0,31 & 0,45 & 0,52 & 0,45 & 0,51 & 0,39 & 0,38 & 0,32 & 0,34 & 0,39 \\
\hline [CINE-P 3] & 0,34 & 0,37 & 0,33 & 0,34 & 0,25 & 0,29 & 0,31 & 0,23 & 0,19 & 0,17 & 0,31 & 0,30 & 0,32 & 0,45 & 0,29 \\
\hline [CINE-P 4-5] & 0,14 & 0,03 & 0,19 & 0,17 & 0,15 & 0,08 & 0,03 & 0,04 & 0,05 & 0,05 & 0,06 & 0,11 & 0,16 & 0,09 & 0,10 \\
\hline [CINE-P 6-8] & 0,09 & 0,14 & 0,24 & 0,17 & 0,12 & 0,26 & 0,16 & 0,03 & 0,19 & 0,20 & 0,15 & 0,18 & 0,12 & 0,11 & 0,16 \\
\hline \multicolumn{16}{|c|}{ Nivel educativo del padre } \\
\hline $\begin{array}{l}\text { No tiene } \\
\text { estudios }\end{array}$ & 0,03 & 0,19 & 0,03 & 0,04 & 0,06 & 0,07 & 0,06 & 0,12 & 0,14 & 0,08 & 0,11 & 0,03 & 0,03 & 0,02 & 0,06 \\
\hline [CINE-P 1-2] & 0,48 & 0,36 & 0,22 & 0,33 & 0,44 & 0,39 & 0,44 & 0,52 & 0,46 & 0,49 & 0,39 & 0,37 & 0,30 & 0,43 & 0,40 \\
\hline [CINE-P 3] & 0,33 & 0,31 & 0,32 & 0,34 & 0,23 & 0,27 & 0,30 & 0,26 & 0,16 & 0,16 & 0,29 & 0,31 & 0,36 & 0,41 & 0,28 \\
\hline [CINE-P 4-5] & 0,07 & 0,02 & 0,14 & 0,13 & 0,14 & 0,10 & 0,05 & 0,04 & 0,06 & 0,04 & 0,06 & 0,11 & 0,16 & 0,06 & 0,09 \\
\hline [CINE-P 6-8] & 0,08 & 0,12 & 0,29 & 0,17 & 0,12 & 0,17 & 0,15 & 0,05 & 0,18 & 0,23 & 0,16 & 0,17 & 0,15 & 0,08 & 0,16 \\
\hline \multicolumn{16}{|c|}{ Tercer grado } \\
\hline \multicolumn{16}{|c|}{ Edad del estudiante } \\
\hline 8 años & 0,18 & 0,00 & 0,05 & 0,13 & 0,00 & 0,18 & 0,33 & 0,01 & 0,09 & 0,03 & 0,16 & 0,17 & 0,22 & 0,03 & 0,11 \\
\hline 9 años & 0,71 & 0,17 & 0,77 & 0,60 & 0,51 & 0,50 & 0,57 & 0,27 & 0,55 & 0,86 & 0,47 & 0,61 & 0,61 & 0,81 & 0,60 \\
\hline 10 a11 años & 0,11 & 0,83 & 0,18 & 0,27 & 0,49 & 0,32 & 0,10 & 0,72 & 0,36 & 0,11 & 0,37 & 0,22 & 0,17 & 0,16 & 0,28 \\
\hline Niña & 0,48 & 0,50 & 0,50 & 0,52 & 0,48 & 0,51 & 0,46 & 0,49 & 0,50 & 0,51 & 0,50 & 0,48 & 0,48 & 0,51 & 0,49 \\
\hline
\end{tabular}


Cuadro A1.4 (conclusión)

\begin{tabular}{|c|c|c|c|c|c|c|c|c|c|c|c|c|c|c|c|}
\hline & ARG & BRA & CHL & COL & CRI & DOM & ECU & GTM & HND & MEX & NIC & PRY & PER & URY & Total \\
\hline Escuela rural & 0,34 & 0,22 & 0,22 & 0,35 & 0,08 & 0,35 & 0,27 & 0,64 & 0,57 & 0,28 & 0,56 & 0,40 & 0,33 & 0,15 & 0,35 \\
\hline $\begin{array}{l}\text { Escuela urbana- } \\
\text { publica }\end{array}$ & 0,40 & 0,46 & 0,18 & 0,22 & 0,72 & 0,38 & 0,48 & 0,25 & 0,11 & 0,44 & 0,22 & 0,23 & 0,35 & 0,64 & 0,36 \\
\hline \multicolumn{16}{|c|}{ Nivel educativo de la madre } \\
\hline $\begin{array}{l}\text { No tiene } \\
\text { estudios }\end{array}$ & 0,02 & 0,14 & 0,02 & 0,03 & 0,06 & 0,08 & 0,05 & 0,16 & 0,13 & 0,06 & 0,10 & 0,04 & 0,08 & 0,01 & 0,06 \\
\hline [CINE-P 1-2] & 0,37 & 0,30 & 0,18 & 0,24 & 0,40 & 0,31 & 0,43 & 0,52 & 0,42 & 0,47 & 0,35 & 0,37 & 0,32 & 0,32 & 0,36 \\
\hline [CINE-P 3] & 0,36 & 0,38 & 0,34 & 0,33 & 0,26 & 0,28 & 0,32 & 0,24 & 0,22 & 0,18 & 0,31 & 0,28 & 0,33 & 0,44 & 0,30 \\
\hline [CINE-P 4-5] & 0,13 & 0,02 & 0,20 & 0,19 & 0,15 & 0,06 & 0,04 & 0,04 & 0,05 & 0,05 & 0,07 & 0,11 & 0,17 & 0,11 & 0,10 \\
\hline [CINE-P 6-8] & 0,11 & 0,16 & 0,25 & 0,20 & 0,13 & 0,27 & 0,15 & 0,04 & 0,19 & 0,23 & 0,17 & 0,20 & 0,11 & 0,11 & 0,17 \\
\hline \multicolumn{16}{|c|}{ Nivel educativo del padre } \\
\hline $\begin{array}{l}\text { No tiene } \\
\text { estudios }\end{array}$ & 0,04 & 0,18 & 0,03 & 0,04 & 0,07 & 0,09 & 0,06 & 0,13 & 0,14 & 0,07 & 0,12 & 0,04 & 0,04 & 0,02 & 0,07 \\
\hline [CINE-P 1-2] & 0,46 & 0,35 & 0,21 & 0,29 & 0,44 & 0,38 & 0,42 & 0,51 & 0,45 & 0,48 & 0,39 & 0,35 & 0,29 & 0,39 & 0,39 \\
\hline [CINE-P 3] & 0,35 & 0,33 & 0,33 & 0,34 & 0,23 & 0,27 & 0,32 & 0,27 & 0,18 & 0,17 & 0,26 & 0,32 & 0,38 & 0,44 & 0,29 \\
\hline [CINE-P 4-5] & 0,07 & 0,02 & 0,15 & 0,15 & 0,14 & 0,08 & 0,06 & 0,05 & 0,07 & 0,05 & 0,07 & 0,11 & 0,16 & 0,06 & 0,09 \\
\hline [CINE-P 6-8] & 0,09 & 0,11 & 0,28 & 0,17 & 0,12 & 0,18 & 0,14 & 0,05 & 0,15 & 0,23 & 0,16 & 0,17 & 0,13 & 0,09 & 0,16 \\
\hline
\end{tabular}

Fuente: Elaboración propia, sobre la base de datos del Tercer Estudio Regional Comparativo y Explicativo (TERCE).

Nota: CINE - Clasificación Internacional Normalizada de la Educación.

Cuadro A1.5

Promedios de las pruebas del Tercer Estudio Regional Comparativo y Explicativo (TERCE) en lectura y matemáticas, sexto y tercer grado

\begin{tabular}{|c|c|c|c|c|c|c|c|c|c|c|c|c|c|c|c|}
\hline & ARG & BRA & $\mathrm{CHL}$ & COL & CRI & DOM & ECU & GTM & HND & MEX & NIC & PRY & PER & URY & Total \\
\hline \multicolumn{16}{|c|}{ Lectura } \\
\hline \multicolumn{16}{|l|}{ Sexto grado } \\
\hline Niña & 723,2 & 746,1 & 795,7 & 743,1 & 769,1 & 651,8 & 693,7 & 684,8 & 702 & 752,5 & 676,4 & 682,8 & 698,8 & 750,2 & 722,5 \\
\hline \multicolumn{16}{|c|}{ Edad del estudiante } \\
\hline 11 años & 714,9 & 711,6 & 763,5 & 748,2 & 806,9 & 662,6 & 693,5 & 709,1 & 703 & 768 & 683,6 & 687,6 & 723,4 & 761,6 & 707,5 \\
\hline 12 años & 720 & 759,4 & 791,2 & 756,3 & 771,8 & 656,6 & 712 & 709,2 & 706,1 & 752,7 & 691,1 & 691,8 & 719,9 & 759,1 & 734,6 \\
\hline 13 años & 668,1 & 752,8 & 776,2 & 725,4 & 767,9 & 629,5 & 664,7 & 694,1 & 701,4 & 747,2 & 658,6 & 634,2 & 643,2 & 680 & 711,9 \\
\hline 14 a15 años & 644,1 & 681,8 & 724,2 & 685,2 & 714,8 & 602,5 & 634,8 & 656,4 & 661,5 & 670,3 & 632,7 & 608,4 & 606,8 & 652 & 652,4 \\
\hline Escuela rural & 679,4 & 682,1 & 750,7 & 696,5 & 734 & 620,4 & 684,2 & 673 & 665,2 & 692,9 & 651,7 & 625,3 & 614,7 & 712,6 & 672,1 \\
\hline $\begin{array}{l}\text { Escuela urbana- } \\
\text { pública }\end{array}$ & 706,6 & 726,2 & 757,2 & 741 & 750,1 & 645,2 & 688,1 & 688,5 & 690,6 & 742,3 & 673,1 & 661,6 & 708,2 & 726,6 & 716,0 \\
\hline \multicolumn{16}{|c|}{ Nivel educativo del padre } \\
\hline $\begin{array}{l}\text { No tiene } \\
\text { estudios }\end{array}$ & 676,6 & 686,9 & 740,5 & 680,6 & 719,9 & 608,7 & 626,1 & 652,3 & 644,4 & 668,2 & 632,4 & 609,7 & 610,7 & 673,6 & 656,1 \\
\hline [CINE-P 1-2] & 689,2 & 725,6 & 745,9 & 700 & 743,1 & 629,3 & 671,1 & 670 & 672,9 & 719,5 & 649,6 & 629,1 & 633,9 & 708,1 & 686,5 \\
\hline [CINE-P 3] & 725,1 & 754,9 & 768,9 & 731 & 767,2 & 643,9 & 711 & 709,7 & 721,5 & 766,2 & 683,2 & 680,1 & 702,7 & 755,9 & 723,4 \\
\hline [CINE-P 4-5] & 733,1 & 745,6 & 797,4 & 770,8 & 802,9 & 661,4 & 739,6 & 735,4 & 741,6 & 792,8 & 700,7 & 714,5 & 757,6 & 794,4 & 756,5 \\
\hline [CINE-P 6] & 746,7 & 805,1 & 826,7 & 802,8 & 807,4 & 675,8 & 759 & 767,6 & 763,9 & 814,8 & 718,4 & 747,5 & 778,7 & 823,2 & 780,8 \\
\hline [CINE-P 7-8] & 799,8 & 820,9 & 844,8 & 835,1 & 835,4 & 698,4 & 785,6 & 795,7 & 773,1 & 827,8 & 740,9 & 752,5 & 822,8 & 850,8 & 806,7 \\
\hline \multicolumn{16}{|c|}{ Nivel educativo de la madre } \\
\hline $\begin{array}{l}\text { No tiene } \\
\text { estudios }\end{array}$ & 676,2 & 682,7 & 742,8 & 666 & 722,7 & 607,1 & 630,7 & 658,2 & 647,4 & 664 & 633 & 606,6 & 607,2 & 667,8 & 654,7 \\
\hline [CINE-P 1-2] & 687,4 & 725,6 & 746,6 & 701,4 & 742,4 & 625,5 & 670 & 676 & 674,1 & 721,1 & 650,1 & 636,4 & 645,9 & 698 & 688,9 \\
\hline [CINE-P 3] & 718,8 & 754,8 & 772,1 & 725,7 & 764,7 & 647,5 & 713,4 & 717,3 & 719,6 & 782,3 & 682,8 & 677,6 & 713,6 & 750,3 & 726,0 \\
\hline [CINE-P 4-5] & 728,5 & 736,3 & 796,4 & 767 & 810,8 & 661,9 & 761 & 760,6 & 738,7 & 791 & 704,6 & 724,6 & 766 & 796,4 & 765,1 \\
\hline [CINE-P 6] & 765,6 & 784,7 & 832,3 & 812,4 & 816,4 & 673,7 & 761,3 & 774,6 & 766,6 & 820,4 & 725,4 & 753,5 & 796,6 & 823,8 & 781,3 \\
\hline [CINE-P 7-8] & 780,7 & 827,3 & 853 & 831,5 & 836,7 & 713,8 & 795,4 & 779,3 & 787,8 & 825,1 & 754,2 & 765,3 & 826,7 & 852,8 & 807,0 \\
\hline
\end{tabular}


Cuadro A1.5 (continuación)

\begin{tabular}{|c|c|c|c|c|c|c|c|c|c|c|c|c|c|c|c|}
\hline & ARG & BRA & CHL & $\mathrm{COL}$ & CRI & DOM & ECU & GTM & HND & MEX & NIC & PRY & PER & URY & Total \\
\hline \multicolumn{16}{|l|}{ Tercer grado } \\
\hline Niña & 721,9 & 736,6 & 814,1 & 741,2 & 767,6 & 639,2 & 707,9 & 701 & 711,6 & 748,5 & 678 & 678,6 & 721,9 & 738,9 & 725,4 \\
\hline \multicolumn{16}{|c|}{ Edad del estudiante } \\
\hline 8 años & 714,4 & 793 & 790,4 & 744,8 & 716,1 & 641,5 & 701,3 & 738,7 & 707,8 & 716,9 & 680,8 & 687,5 & 733,6 & 709,8 & 708,7 \\
\hline 9 años & 719,1 & 752 & 809,7 & 745,8 & 761,6 & 645,7 & 717,3 & 712,9 & 709,2 & 742 & 687,1 & 683,3 & 733,3 & 740,1 & 731,7 \\
\hline 10 años & 660 & 740,2 & 809,2 & 722 & 768,6 & 603,1 & 690,8 & 713,4 & 714 & 745,2 & 652,8 & 620,6 & 653,9 & 681 & 713,7 \\
\hline 11 años & 640,3 & 675,3 & 763,6 & 671,7 & 704,5 & 606,7 & 656,3 & 663,4 & 675,8 & 662,5 & 644,8 & 627,8 & 645,4 & 660,5 & 658,6 \\
\hline Escuela rural & 691,2 & 688,3 & 773 & 689,1 & 727,5 & 614,4 & 691,4 & 680,6 & 675,7 & 693,9 & 651,1 & 632,1 & 647,8 & 709 & 677,0 \\
\hline $\begin{array}{l}\text { Escuela urbana- } \\
\text { pública }\end{array}$ & 700,1 & 715,8 & 778,5 & 727 & 747,2 & 621,7 & 702,1 & 706,4 & 702,1 & 724,9 & 672,8 & 662,9 & 727,2 & 713,7 & 713,4 \\
\hline \multicolumn{16}{|c|}{ Nivel educativo del padre } \\
\hline $\begin{array}{l}\text { No tiene } \\
\text { estudios }\end{array}$ & 684,4 & 675,3 & 748,8 & 663,4 & 714,8 & 605,6 & 660,8 & 658 & 662,1 & 663,2 & 644,7 & 601,9 & 645 & 680,1 & 661,2 \\
\hline [CINE-P 1-2] & 691,1 & 717,1 & 769,8 & 696,4 & 741,1 & 616,2 & 683,9 & 678,6 & 684,1 & 711,1 & 652,9 & 637,3 & 661,2 & 695,7 & 688,4 \\
\hline [CINE-P 3] & 721,4 & 745,8 & 793,9 & 728 & 756,4 & 625,2 & 716,9 & 729,2 & 724 & 755,9 & 671,9 & 674,1 & 721,2 & 741,6 & 724,2 \\
\hline [CINE-P 4-5] & 746,8 & 768,6 & 817,8 & 762,9 & 794,7 & 657,9 & 750 & 754,1 & 730,4 & 763,4 & 704,3 & 695,6 & 774,8 & 774,5 & 758,6 \\
\hline [CINE-P 6] & 756,6 & 787,4 & 847,1 & 799,7 & 814,1 & 674 & 761,1 & 785,1 & 774,7 & 804,9 & 722,1 & 737,6 & 791,5 & 790,5 & 780,0 \\
\hline [CINE-P 7-8] & 764,8 & 808,3 & 865,9 & 823,9 & 830,5 & 696,5 & 789 & 793,5 & 793,3 & 823,1 & 738,7 & 725,7 & 813,3 & 815,8 & 809,7 \\
\hline \multicolumn{16}{|c|}{ Nivel educativo de la madre } \\
\hline $\begin{array}{l}\text { No tiene } \\
\text { estudios }\end{array}$ & 678,7 & 667,1 & 758,2 & 664,1 & 702,2 & 604 & 663 & 660,1 & 662,7 & 659,9 & 641,7 & 604,7 & 637,3 & 704,7 & 658,6 \\
\hline [CINE-P 1-2] & 684,5 & 708,6 & 767,6 & 686,5 & 738,4 & 611,2 & 682,7 & 688 & 684,3 & 712,8 & 656 & 638,5 & 669,3 & 688,8 & 688,9 \\
\hline [CINE-P 3] & 720,5 & 742,3 & 795,8 & 728,2 & 766,5 & 627 & 718,9 & 737,7 & 738,1 & 764,1 & 670 & 669,3 & 735,2 & 738 & 728,6 \\
\hline [CINE-P 4-5] & 732,4 & 752,5 & 817,8 & 766,5 & 790,2 & 651,5 & 752,3 & 783,3 & 739,5 & 767,6 & 697,3 & 707 & 781,1 & 768,9 & 765,7 \\
\hline [CINE-P 6] & 750,7 & 793,4 & 852,1 & 796,1 & 810,5 & 671,2 & 760,8 & 798,1 & 765,7 & 802,4 & 733,3 & 740 & 809,4 & 795 & 777,5 \\
\hline [CINE-P 7-8] & 771,3 & 803,5 & 873,6 & 823,7 & 825,6 & 719,4 & 791,9 & 803,7 & 777,9 & 834,6 & 745 & 755,8 & 835,7 & 799,5 & 811,9 \\
\hline \multicolumn{16}{|c|}{ Matemáticas } \\
\hline \multicolumn{16}{|l|}{ Sexto grado } \\
\hline Niña & 721,4 & 720,8 & 802,2 & 707,7 & 737,5 & 633,8 & 700,6 & 672,8 & 685,4 & 771 & 650,1 & 657,4 & 707,6 & 767,2 & 713,8 \\
\hline \multicolumn{16}{|c|}{ Edad del estudiante } \\
\hline 11 años & 723,4 & 711,6 & 782,6 & 722,7 & 759,9 & 645,9 & 706,1 & 694,7 & 700,7 & 785,2 & 660,3 & 674,7 & 737 & 791,6 & 707,8 \\
\hline 12 años & 732 & 745 & 808,7 & 728,1 & 749,8 & 642,4 & 715,6 & 703,5 & 693,4 & 783,8 & 670,4 & 671,7 & 735,5 & 784,1 & 736,3 \\
\hline 13 años & 690,7 & 740,2 & 801,3 & 708,2 & 745,4 & 625,7 & 683,5 & 687,1 & 694,3 & 774,5 & 637,1 & 633 & 661,8 & 713,7 & 712,2 \\
\hline 14 a 15 años & 673,6 & 677,5 & 732,7 & 676,7 & 696,6 & 611,8 & 662,5 & 657,7 & 663,3 & 688,4 & 636,1 & 620,2 & 643 & 679,1 & 657,6 \\
\hline Escuela rural & 700,9 & 681,5 & 762,2 & 682,4 & 707,5 & 620,4 & 691,9 & 671,6 & 666,1 & 732,6 & 640,8 & 633,5 & 644,8 & 750,8 & 677,2 \\
\hline $\begin{array}{l}\text { Escuela urbana- } \\
\text { pública }\end{array}$ & 721,7 & 713,1 & 767,2 & 710,4 & 729,7 & 635,8 & 700,7 & 679,9 & 667,1 & 772,3 & 651 & 644 & 721 & 754,2 & 719,0 \\
\hline \multicolumn{16}{|c|}{ Nivel educativo del padre } \\
\hline $\begin{array}{l}\text { No tiene } \\
\text { estudios }\end{array}$ & 699,6 & 680 & 744,2 & 665,2 & 701,5 & 618,5 & 661,3 & 657,6 & 649,1 & 707,2 & 631,7 & 624,9 & 639,4 & 686,5 & 663,7 \\
\hline [CINE-P 1-2] & 705,9 & 716,8 & 757,6 & 682,1 & 723,7 & 625,3 & 687 & 670,1 & 670,5 & 753,4 & 639,9 & 632,7 & 661,3 & 737,9 & 693,8 \\
\hline [CINE-P 3] & 734,5 & 737,5 & 781,7 & 708,9 & 740,9 & 630,5 & 714,7 & 696,1 & 708,1 & 790,7 & 659,8 & 660,5 & 716,3 & 784,1 & 719,8 \\
\hline [CINE-P 4-5] & 751 & 742,3 & 821,4 & 740,4 & 779,6 & 646,6 & 735,4 & 724,9 & 719 & 804,9 & 677,2 & 685 & 768,9 & 811,2 & 752,3 \\
\hline [CINE-P 6] & 753,5 & 793,3 & 853,9 & 775,9 & 781,9 & 660,2 & 752 & 750,4 & 738,6 & 839,2 & 690,5 & 717,2 & 794,8 & 840,4 & 778,4 \\
\hline [CINE-P 7-8] & 805,7 & 820,6 & 876,3 & 811,3 & 813,9 & 679,6 & 782,3 & 771,7 & 756,7 & 849,4 & 701,5 & 711,1 & 815,1 & 850,4 & 803,7 \\
\hline
\end{tabular}


Cuadro A1.5 (conclusión)

\begin{tabular}{|c|c|c|c|c|c|c|c|c|c|c|c|c|c|c|c|}
\hline & ARG & BRA & CHL & $\mathrm{COL}$ & CRI & DOM & ECU & GTM & HND & MEX & NIC & PRY & PER & URY & Total \\
\hline \multicolumn{16}{|c|}{ Nivel educativo de la madre } \\
\hline $\begin{array}{l}\text { No tiene } \\
\text { estudios }\end{array}$ & 702,2 & 682,5 & 760,5 & 666,2 & 704,6 & 25,2 & 652,1 & 660,8 & 645 & 710,2 & 638,5 & 612,1 & 635 & 698,9 & 665,0 \\
\hline [CINE-P 1-2] & 701,6 & 711,9 & 756,4 & 682,7 & 723,9 & 623,8 & 688 & 672 & 672,1 & 754,8 & 640 & 638,6 & 671,9 & 727 & 696,0 \\
\hline [CINE-P 3] & 727,7 & 737,7 & 786,4 & 704,3 & 741 & 632,9 & 717,4 & 709,1 & 709 & 803 & 658,3 & 660,1 & 726 & 777,6 & 723,3 \\
\hline [CINE-P 4- & 755,6 & 734,9 & 822,1 & 740,1 & 785,7 & 644,3 & 750 & 744,2 & 725,2 & 807 & 675,8 & 689,1 & 778,4 & 811,9 & 765,0 \\
\hline [CINE-P 6] & 769,5 & 783,5 & 859,4 & 781 & 791 & 655,1 & 753,5 & 757,4 & 741,8 & 838,9 & 698,9 & 721,2 & 807,9 & 850,6 & 775,6 \\
\hline [CINE- $\mathrm{F}$ & 786,8 & 826,7 & 881,4 & 805,8 & 811,4 & 674 & 782,6 & 744,5 & 754,9 & 855,2 & 717,2 & 720,8 & 821,5 & 852,8 & 794,9 \\
\hline \multicolumn{16}{|l|}{ Tercer grado } \\
\hline Niña & 725,8 & 747,9 & 796,7 & 714,6 & 754,4 & 629,3 & 701,6 & 689,1 & 706,5 & 758,3 & 668 & 672,1 & 712,3 & 748,9 & 719,2 \\
\hline \multicolumn{16}{|c|}{ Edad del estudiante } \\
\hline 8 años & 731,9 & 753,4 & 782,1 & 723,5 & 699,7 & 636,8 & 697,8 & 708,1 & 709,8 & 730 & 675,5 & 676,2 & 730,9 & 727,8 & 705,5 \\
\hline 9 años & 729,9 & 770,8 & 799,1 & 727,4 & 759,1 & 636,8 & 709,1 & 705,8 & 707,7 & 759,8 & 680,1 & 683,7 & 731,1 & 757,6 & 732,2 \\
\hline 10 años & 674,4 & 759 & 795,3 & 708,4 & 764,8 & 602 & 688,4 & 708,7 & 713 & 746,9 & 653,8 & 638 & 655,5 & 685 & 714,9 \\
\hline 11 años & 648,3 & 691,3 & 739,4 & 660,8 & 708,5 & 606,3 & 665,4 & 656,8 & 681,9 & 681,9 & 652,6 & 623,5 & 645,3 & 657,4 & 659,7 \\
\hline Escuela rural & 704,1 & 707,7 & 761,3 & 675,7 & 724,3 & 610,8 & 687,5 & 675,8 & 681,3 & 717,8 & 656,3 & 649,5 & 647,8 & 726,9 & 679,9 \\
\hline $\begin{array}{l}\text { Escuela urbana } \\
\text { pública }\end{array}$ & 712,3 & 730,6 & 766,2 & 706,8 & 745,2 & 613,8 & 698,7 & 698,8 & 691,3 & 745,4 & 663,8 & 654,3 & 724,8 & 726,1 & 716,0 \\
\hline \multicolumn{16}{|c|}{ Nivel educativo del padre } \\
\hline $\begin{array}{l}\text { No tiene } \\
\text { estudios }\end{array}$ & 679,1 & 694 & 742,9 & 652 & 707,8 & 608 & 673,6 & 647,5 & 667,1 & 690,4 & 654,8 & 621,6 & 629,2 & 691,7 & 665,7 \\
\hline [CINE-P 1-2] & 706,5 & 732,6 & 757,2 & 679,6 & 738,5 & 609,3 & 682,9 & 673,7 & 687,8 & 732,5 & 656,6 & 648,2 & 660,3 & 706,7 & 692,4 \\
\hline [CINE-P 3] & 733,4 & 761,1 & 780,9 & 712,1 & 757,7 & 624 & 711,9 & 722,1 & 721,9 & 770,1 & 661,2 & 667,4 & 720 & 758,9 & 723,2 \\
\hline [CINE-P 4-5] & 749,8 & 798,1 & 810,1 & 744,6 & 786,8 & 655,2 & 722,5 & 748,4 & 729,7 & 774,1 & 687,4 & 690,6 & 774 & 789,4 & 753,4 \\
\hline [CINE-P 6] & 767,5 & 820 & 836,7 & 776,3 & 811,2 & 660,4 & 739 & 779,5 & 763,1 & 810,5 & 712,1 & 726,4 & 790,8 & 815,4 & 775,3 \\
\hline [CINE-P 7-8] & 773,1 & 829,6 & 855,3 & 816,7 & 831,2 & 670,5 & 770,2 & 790,6 & 772,8 & 822,4 & 742,6 & 716,6 & 801,6 & 836,3 & 803,8 \\
\hline \multicolumn{16}{|c|}{ Nivel educativo de la madre } \\
\hline $\begin{array}{l}\text { No tiene } \\
\text { estudios }\end{array}$ & 692,1 & 684,2 & 748,6 & 646,7 & 700,9 & 609,5 & 673,7 & 652,3 & 671,5 & 682,9 & 657,7 & 611,5 & 641 & 695,2 & 663,4 \\
\hline [CINE-P 1-2] & 701,9 & 727,6 & 755,1 & 672,2 & 739 & 608 & 682,4 & 680,7 & 687 & 734,8 & 654,1 & 651,8 & 669,9 & 699,3 & 693,7 \\
\hline [CINE-P 3] & 728,2 & 758,3 & 781,7 & 711,5 & 761,8 & 624 & 713,7 & 733,2 & 732,9 & 779,1 & 666,8 & 667 & 733,9 & 752,5 & 727,7 \\
\hline [CINE-P 4-5] & 745,7 & 775,2 & 810,4 & 747,4 & 789,2 & 638,9 & 725,8 & 770,3 & 740,5 & 782 & 693,7 & 694,3 & 778,6 & 791,3 & 762,5 \\
\hline [CINE-P 6] & 764 & 822,8 & 846 & 773 & 801,2 & 659,6 & 739,5 & 791,4 & 754,7 & 809,8 & 719 & 727,6 & 805,7 & 820,7 & 772,1 \\
\hline [CINE-P 7-8] & 806,4 & 809,7 & 850,1 & 805,4 & 823,5 & 708,3 & 772,9 & 800 & 765,9 & 820,8 & 733,7 & 731,6 & 817,8 & 823,6 & 801,0 \\
\hline
\end{tabular}

Fuente: Elaboración propia, sobre la base de datos del Tercer Estudio Regional Comparativo y Explicativo (TERCE). 